\title{
Global transport in a nonautonomous periodic standard map
}

\author{
R. Calleja, ${ }^{1, a)}$ D. del-Castillo-Negrete, ${ }^{2, b)}$ D. Martínez-del-Río, ${ }^{1, c)}$ and A. Olvera ${ }^{1, d)}$ \\ 1) IIMAS-UNAM, Mexico D.F., 04510, Mexico \\ ${ }^{2)}$ Oak Ridge National Laboratory, Oak Ridge, Tennessee, 37831-8071, \\ $U S A$
}

A non-autonomous version of the standard map with a periodic variation of the perturbation parameter is introduced and studied via an autonomous map obtained from the iteration of the nonautonomous map over a period. Symmetry properties in the variables and parameters of the map are found and used to find relations between rotation numbers of invariant sets. The role of the nonautonomous dynamics on period-one orbits, stability and bifurcation is studied. The critical boundaries for the global transport and for the destruction of invariant circles with fixed rotation number are studied in detail using direct computation and a continuation method. In the case of global transport, the critical boundary has a particular symmetrical horn shape. The results are contrasted with similar calculations found in the literature.

\footnotetext{
a)Electronic mail: calleja@mym.iimas.unam.mx

b) Electronic mail: delcastillo@ornl.gov

c) Electronic mail: dmr@mym.iimas.unam.mx

d)Electronic mail: aoc@mym.iimas.unam.mx
} 


\section{INTRODUCTION}

Two dimensional maps have been extensively studied within discrete dynamical systems ${ }^{35}$. However, studies of non-autonomous maps seem to be scarce, even in the two dimensional

case. Here the term non-autonomous is used to describe maps, $T: \mathrm{x}_{n} \mapsto \mathrm{x}_{n+1}$, that exhibit an explicit dependence on the iteration number, $n$, i.e., $\mathbf{x}_{n+1}=f\left(\mathbf{x}_{n}, n\right)$.

In this paper we present a detailed numerical study of a non-autonomous standard map (NASM) in which the perturbation parameter exhibits a periodic dependence. In Ref. 36 it was shown that this map naturally arises in the study of mean-field coupled symplectic maps introduced in Refs. 37-39 as simplified models of self-consistent chaos in many-degrees-of freedom systems.

To give a physics interpretation and motivation of the NASM, recall that the standard map, $x_{n+1}=x_{n}+y_{n+1}, y_{n+1}=y_{n}+\frac{\kappa}{2 \pi} \sin \left(2 \pi x_{n}\right)$, provides a simple model to study the chaotic dynamics of particles subject to periodic forces. An example is the motion of charged particles in the presence of electrostatic waves whose amplitudes (assumed to be fixed) determine the perturbation amplitude $\kappa$. A natural extension of this highly simplified description is the relaxation of the strong assumption that the waves' amplitudes remain fixed, i.e. to make the map non-autonomous by allowing the perturbation parameter to depend on the iteration $n$. Although in principle $\kappa_{n}$ can exhibit a wide range of dynamical behaviors (e.g, periodic, quasi-periodic, or random) the case of periodic dependence ${ }^{36}$ is particularly relevant in the nonlinear saturation of the wave-particle interaction. For example, numerical and analytical results have shown that the self-consistent dynamics of electrons in the beam-plasma instability generically lead to the formation of phase-space coherent structures accompanied by the self-consistent, approximately time-periodic, dependence of the electrostatic field's amplitude $^{37,40}$. Beyond plasma physics, there are other areas in which the NASM could be of relevance. For example, as discussed in Refs. 37 and 39, mean-field coupled symplectic maps can also be applied to the study of vortex dynamics and transport in strong shear flows. Based on this, the NASM could also be applied to the study of chaotic advection and mixing in fluids exhibiting wave dynamics with time-periodic evolution.

Previous studies related to the work discussed here include Refs. 41-44 that considered maps constructed from the composition of twist maps. Reference 41 considered the convergence to asymptotic invariant tori in families of non-autonomous maps that converge to 
a prescribed autonomous map with known invariant tori. On the other hand, the rotating standard map in Ref. 42 and the driven standard map in Ref. 43 can be considered generalizations of the NASM, although with different aims: a search of the bifurcation space where the two dimensional tori cease to exist and regions of stability of invariant curves in the parameter space, respectively. Reference 44 studies the case of random iterations of an arbitrary number of two dimensional maps which might or might not be twist.

Here we focus on the case when the perturbation parameter exhibits period-two behavior, i.e., $\left\{\kappa_{n}\right\}=\left\{\kappa_{1}, \kappa_{2}, \kappa_{1}, \ldots\right\}$. In this case, the NASM can be formulated as the composition of two standard maps (with perturbation parameters $\kappa_{1}$ and $\kappa_{2}$ ) that results in an autonomous, albeit complicated, map which incidentally might not satisfy the twist condition (which is typically assumed in studies of symplectic maps). Our main goal is to characterize the regions in the $\left(\kappa_{1}, \kappa_{2}\right)$ parameter space for which there is global chaos, i.e. the regions where the composed map has no KAM (Kolmogorov-Arnold-Moser) invariant circles. From the physics point of view this corresponds to parameters for which there are initial conditions that exhibit unbounded trajectories in $y$.

The rest of the paper is organized as follows. Section II defines the NASM and discusses its basic properties. In Sec. III, we study the NASM symmetries in the coordinates and parameters spaces, and present relations between rotation numbers of invariant circles for symmetric values of the parameters. In section IV, we study low-order periodic orbits of the NASM. In Sec. V, we study some reduced cases of the map and give a criteria to search numerically for the threshold of global transport. In Sec. VI, we search again for transport barriers as function of the parameters, using the parameterization method, applied on particular interesting rotation numbers. In Sec. VII, we compare these results with previous works in Refs. 41-43 and propose some conjectures and present the conclusions.

\section{MAP DEFINITION.}

Our starting point is the nonautonomous standard map (NASM), defined as

$$
\begin{aligned}
& \hat{x}_{n+1}=\hat{x}_{n}+\hat{y}_{n+1} \quad \bmod (1), \\
& \hat{y}_{n+1}=\hat{y}_{n}+\frac{\kappa_{n}}{2 \pi} \sin \left(2 \pi \hat{x}_{n}\right) .
\end{aligned}
$$

where $\kappa_{n}$ is a function of $n$. 
Motivated by the asymptotic dynamics of self-consistent coupled maps ${ }^{36,37}$, we focus on the case when $\kappa_{n}$ is a periodic function. The periodic case always allows to give an autonomous representation of the nonautonomous map in Eq. (1) as the consecutive iteration of a whole period. And on top of that, a high period variation of $\kappa_{n}$ would allow to mimic observed data from more complex models and establish hierarchy relations between the parameters. However we have encountered evidence that suggest that the increment of only one parameter cause a transcendental change in the critical global transport problem ${ }^{36,37}$. The properties and relations between the nonautonomous map (1) and the autonomous version are presented for the simplest periodic case in this section.

As a first step, we consider a "triangular wave" periodic dependence in which $\kappa_{n}$ can only take two values:

$$
\kappa_{n}= \begin{cases}\kappa_{1} & \text { if } n \text { is odd } \\ \kappa_{2} & \text { if } n \text { is even. }\end{cases}
$$

We define a new map, $\mathcal{T}_{\kappa_{1} \kappa_{2}}$, such that its iterates $\left(x_{n}, y_{n}\right) n=1,2, \ldots$ coincide with the even iterations of (1), i.e.,

$$
\left(x_{n}, y_{n}\right)=\left(\hat{x}_{2 n}, \hat{x}_{2 n}\right) .
$$

By construction, the map $\mathcal{T}_{\kappa_{1} \kappa_{2}}$ is autonomous and can be written as,

$$
\begin{aligned}
& x_{n+1}=x_{n}+2 y_{n}+\mathcal{F}_{1}\left(x_{n}, y_{n} ; \kappa_{1}, \kappa_{2}\right) \quad \bmod (1) \\
& y_{n+1}=y_{n}+\mathcal{F}_{2}\left(x_{n}, y_{n} ; \kappa_{1}, \kappa_{2}\right)
\end{aligned}
$$

where the functions $\mathcal{F}_{1}$ and $\mathcal{F}_{2}$ are defined as,

$$
\begin{aligned}
\mathcal{F}_{1}\left(x, y ; \kappa_{1}, \kappa_{2}\right) & =\frac{\kappa_{1}}{2 \pi} \sin (2 \pi x)+\mathcal{F}_{2}, \\
\mathcal{F}_{2}\left(x, y ; \kappa_{1}, \kappa_{2}\right) & =\frac{\kappa_{1}}{2 \pi} \sin (2 \pi x) \\
+\frac{\kappa_{2}}{2 \pi} \sin & \left\{2 \pi\left[x+y+\frac{\kappa_{1}}{2 \pi} \sin (2 \pi x)\right]\right\} .
\end{aligned}
$$

It should be noted that this new autonomous map preserves orientation and depends of an infinite number of harmonics when the term $\sin [a+\sin (b)]$ is expanded in Bessel functions and because of this, the map is not reducible to the extensively studied Refs. 45-48 two frequency standard map.

As Fig. 1(c) shows, due to its non autonomous nature, the map in (1) exhibits selfintersection of trajectories, something that as Fig. 1(a) and Fig. 1(b) illustrates, never 
happens in autonomous maps. Fig. 1(d) shows, the non autonomous dynamics of an initial condition inside an island of (1) alternates between $(0,1 / 2)$ elliptic point of $\mathcal{T}_{\kappa_{1} \kappa_{2}}$ and $(1 / 2,1 / 2)$ elliptic point of $\mathcal{T}_{\kappa_{2} \kappa_{1}}$.

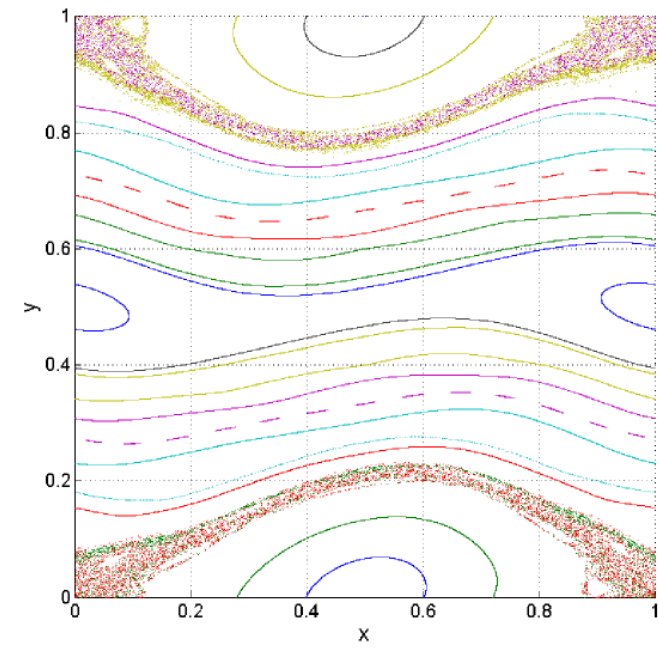

(a)

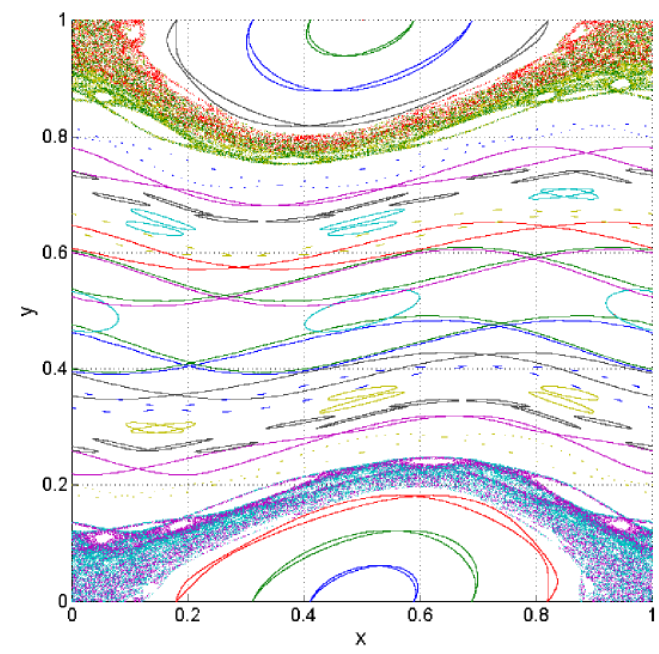

(c)

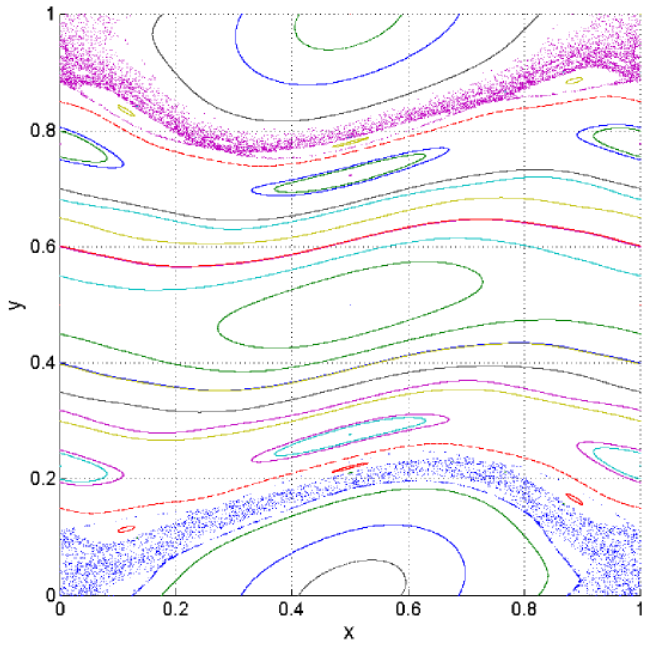

(b)

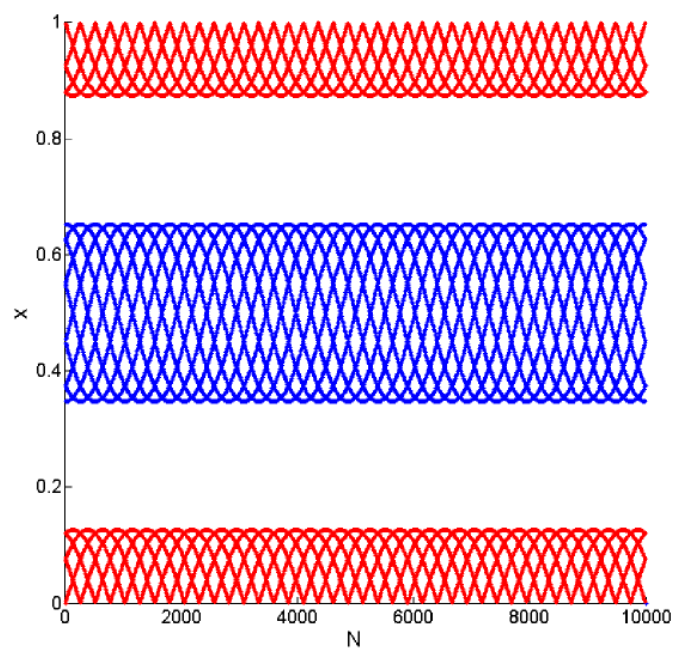

(d)

Figure 1. Comparison between the phase space of maps (4) and (1). (a) and (b) correspond to (4) with $\left(\kappa_{1}, \kappa_{2}\right):(0.35,0.5)$ and $(0.5,0.35)$ respectively, and (c) corresponds to map (1) with the same parameters. (d) shows to the time series of the initial condition $z_{0}=(0,0.55)$, where blue (red) corresponds to the even (odd) iterates of map (1).

The map in Eq. (4) is equivalent to the composition of two standard maps, i.e., $\mathcal{T}_{\kappa_{1} \kappa_{2}} \equiv$ 
$\mathcal{S}_{\kappa_{2}} \circ \mathcal{S}_{\kappa_{1}}$, where $\mathcal{S}_{\epsilon}$ denotes the standard map with perturbation parameter $\epsilon$,

$$
\mathcal{S}_{\epsilon}\left(\begin{array}{l}
x \\
y
\end{array}\right)=\left(\begin{array}{c}
x+y+\frac{\epsilon}{2 \pi} \sin (2 \pi x) \\
y+\frac{\epsilon}{2 \pi} \sin (2 \pi x)
\end{array}\right) \text {. }
$$

with $(x, y) \in \mathbb{T} \times \mathbb{R}$. The composition of (positive) twist maps is denoted as positive tilt map $^{49}$ in the literature. Although, tilt maps share many features with twist maps it is easy to see that in general tilt maps in general are not twist. It is straightforward to show that $\mathcal{T}_{\kappa_{1} \kappa_{2}}$ and $\mathcal{T}_{\kappa_{2} \kappa_{1}}$ are diffeomorphic, since,

$$
\mathcal{S}_{\kappa_{2}}^{-1} \circ \mathcal{T}_{\kappa_{1} \kappa_{2}}^{n} \circ \mathcal{S}_{\kappa_{2}}=\mathcal{T}_{\kappa_{2} \kappa_{1}}^{n}
$$

where $\mathcal{S}_{\epsilon}^{-1}$ denotes the inverse of the standard map,

$$
\mathcal{S}_{\epsilon}^{-1}\left(\begin{array}{l}
x \\
y
\end{array}\right)=\left(\begin{array}{c}
x-y \\
y-\frac{\epsilon}{2 \pi} \sin (2 \pi(x-y))
\end{array}\right) .
$$

Note that, as shown in Fig. 1, the two autonomous representations have the same kind of invariant sets. This important remark, that results from the conjugacy in Eq. (7), is discussed in Secs. III B and VIA where it is proved that if an invariant circle exists in one of the representations then an invariant circle with the same rotation number exists in the other representation.

This itself implies that if there exist an invariant circle in any of the autonomous representations, then there exists a barrier for the transport in the nonautonomous map (1), since the autonomous representations are also stroboscopic maps of (1). By invariance, the invariant circles of $\mathcal{T}_{\kappa_{1} \kappa_{2}}$ and $\mathcal{T}_{\kappa_{2} \kappa_{1}}$ limit the vertical displacement (drift) of any orbit in the map, so, if there is no unbounded drift in any of the stroboscopic maps, there is not for the whole map, even if in general there is not a defined invariant circle $^{44}$ for map (1).

The twist of $\mathcal{T}_{\kappa_{1} \kappa_{2}}$ is given by,

$$
\left.\frac{\partial x_{n+1}}{\partial y_{n}}\right|_{x_{n}}=2+\kappa_{2} \cos \left\{2 \pi\left[x_{n}+y_{n}+\frac{\kappa_{1}}{2 \pi} \sin \left(2 \pi x_{n}\right)\right]\right\} .
$$

and the twist condition $\left.\frac{\partial x_{n+1}}{\partial y_{n}}\right|_{x_{n}}>0$, is satisfied in the whole cylinder when $\left|\kappa_{2}\right|<2$. Based on this, we define the twist region in the parameter space as the square: $\left\{\left(\kappa_{1}, \kappa_{2}\right):\left|\kappa_{i}\right|<\right.$ $2, i=1,2\}$, where both $\mathcal{T}_{\kappa_{1} \kappa_{2}}$ and $\mathcal{T}_{\kappa_{2} \kappa_{1}}$ are twist. It must be noted that, if the map is twist for small values of the parameters, the Moser's Twist Theorem ${ }^{50}$ guarantees the existence of invariant circles well ordered with the rotation number. 
Let $\Omega=d y \wedge d x$ be a symplectic form on the cylinder, then it is clear that $\mathcal{T}_{\kappa_{1} \kappa_{2}}$ is symplectic with respect to $\Omega$ since each one of the standard maps is symplectic. In particular,

$$
\left(\mathcal{T}_{\kappa_{1} \kappa_{2}}\right)^{*} \Omega=\left(\mathcal{S}_{\kappa_{2}} \circ \mathcal{S}_{\kappa_{1}}\right)^{*} \Omega=\mathcal{S}_{\kappa_{1}}^{*} \mathcal{S}_{\kappa_{2}}^{*} \Omega=\Omega
$$

where $(\mathrm{F})^{*}$ is the pullback via a function $\mathrm{F}$. We also note that since both $\mathcal{S}_{\kappa_{2}}$ and $\mathcal{S}_{\kappa_{2}}$ are exact, then $\mathcal{T}_{\kappa_{1} \kappa_{2}}$ is also exact. Notice that if $d \alpha=\Omega$, then

$$
\mathcal{S}_{\kappa_{i}}^{*} \alpha-\alpha=d P_{i} \quad i=1,2
$$

where $P_{i}$ is the generating functions of $\mathcal{S}_{\kappa_{i}}$. The simple computation is as follows (see Ref. 51).

$$
\begin{aligned}
\mathcal{T}_{\kappa_{1} \kappa_{2}}^{*} \alpha & =\left(\mathcal{S}_{\kappa_{2}} \circ \mathcal{S}_{\kappa_{1}}\right)^{*} \alpha=\mathcal{S}_{\kappa_{1}}^{*} \mathcal{S}_{\kappa_{2}}^{*} \alpha=\mathcal{S}_{\kappa_{1}}^{*}\left(\alpha+d P_{2}\right) \\
& =\alpha+d\left(P_{1}+\mathcal{S}_{\kappa_{1}}^{*} P_{2}\right)
\end{aligned}
$$

Therefore $\mathcal{T}_{\kappa_{1} \kappa_{2}}$ is exact with generating function $P=P_{1}+\mathcal{S}_{\kappa_{1}}^{*} P_{2}$. Using Mather's conjunction operation ${ }^{52}$ it is possible to give an implicit expression for this generating function $P$ as,

$$
P=P_{1} * P_{2}
$$

where $*$ stands for,

$$
P_{1} * P_{2}\left(x, x^{\prime}\right)=\min _{\xi}\left(P_{1}(x, \xi)+P_{2}\left(\xi, x^{\prime}\right)\right) .
$$

In our case, the $\xi$ that minimizes the conjunction is the unique solution to the equation,

$$
\xi=\frac{x+x^{\prime}}{2}+\frac{\kappa_{2}}{4 \pi} \sin (2 \pi \xi)
$$

And this last expression has clearly only one solution whenever $\left|\kappa_{2}\right|<2$, which as mentioned before corresponds to the region in the parameter space where the twist condition is satisfied.

\section{SYMMETRIES.}

The symmetries of the NASM, help to reduce different possible cases and to infer the existence of invariant circles and their rotation numbers from the existence of invariant circle with different parameter values. Since the autonomous representation of the NASM in (4) is the composition of two standard maps (6), we expect to have the symmetries of the standard map. However there are other symmetries of $\mathcal{T}_{\kappa_{1} \kappa_{2}}$. 
The functions $\mathcal{F}_{i}$ in Eqs. (5a) and (5b) have the following symmetries.

$$
\begin{array}{ll}
\text { P1. } & \mathcal{F}_{i}\left(-x,-y ; \kappa_{1}, \kappa_{2}\right)=-\mathcal{F}_{i}\left(x, y ; \kappa_{1}, \kappa_{2}\right) . \\
\text { P2. } & \mathcal{F}_{i}\left(x+n, y+m ; \kappa_{1}, \kappa_{2}\right)=\mathcal{F}_{i}\left(x, y ; \kappa_{1}, \kappa_{2}\right), \\
& \text { for } n, m \in \mathbb{Z} . \\
\text { P3. } & \mathcal{F}_{i}\left(x, y ; \kappa_{1} ; \kappa_{2}\right)=\mathcal{F}_{i}\left(x+1 / 2, y ;-\kappa_{1},-\kappa_{2}\right) . \\
\text { P4. } & \mathcal{F}_{i}\left(x, y ; \kappa_{1} ; \kappa_{2}\right)=\mathcal{F}_{i}\left(x, y+1 / 2 ; \kappa_{1},-\kappa_{2}\right) .
\end{array}
$$

Whereas Eqs. (15) and (16) are coordinate symmetries directly inherented from the standard map, Eqs. (17) and (18) are symmetries of the non-autonomous map that involve both coordinates and parameter transformations. From, (17) and (18) it follows that,

$$
\mathcal{F}_{i}\left(x, y ; \kappa_{1} ; \kappa_{2}\right)=\mathcal{F}_{i}\left(x+1 / 2, y+1 / 2 ;-\kappa_{1} ; \kappa_{2}\right)
$$

In the remaining of this section, we denote by $(x, y)$ the variables of the lift of map (4). In other words, $x \in \mathbb{R}$.

\section{A. Orbit symmetries.}

Let $x_{n}\left(x_{0}, y_{0} ; \kappa_{1}, \kappa_{2}\right)$ and $y_{n}\left(x_{0}, y_{0} ; \kappa_{1}, \kappa_{2}\right)$ denote the $x$ and $y$ coordinates of the $n$-th iterate of the NASM with parameters $\left(\kappa_{1}, \kappa_{2}\right)$ and initial condition $\left(x_{0}, y_{0}\right)$. Then, from the properties of $\mathcal{F}_{i}$, it follows that the orbits exhibit the following symmetries (see Fig. 2).

1. Coordinate reflection [from Eq. (15)],

$$
\begin{aligned}
& x_{n}\left(-x_{0},-y_{0} ; \kappa_{1}, \kappa_{2}\right)=-x_{n}\left(x_{0}, y_{0} ; \kappa_{1}, \kappa_{2}\right), \\
& y_{n}\left(-x_{0},-y_{0} ; \kappa_{1}, \kappa_{2}\right)=-y_{n}\left(x_{0}, y_{0} ; \kappa_{1}, \kappa_{2}\right) .
\end{aligned}
$$

2. Coordinate translation [from Eq. (16)],

$$
\begin{aligned}
& x_{n}\left(x_{0}+r, y_{0}+s ; \kappa_{1}, \kappa_{2}\right)=x_{n}\left(x_{0}, y_{0} ; \kappa_{1}, \kappa_{2}\right)+r+2 n s, \\
& y_{n}\left(x_{0}+r, y_{0}+s ; \kappa_{1}, \kappa_{2}\right)=y_{n}\left(x_{0}, y_{0} ; \kappa_{1}, \kappa_{2}\right)+s
\end{aligned}
$$

for $r, s \in \mathbb{Z}$. 
3. Coordinate translation and reflection [from Eqs. (20)-(21)],

$$
\begin{aligned}
& x_{n}\left(1-x_{0}, 1-y_{0} ; \kappa_{1}, \kappa_{2}\right)=1-x_{n}\left(x_{0}, y_{0} ; \kappa_{1}, \kappa_{2}\right)+2 n \\
& y_{n}\left(1-x_{0}, 1-y_{0} ; \kappa_{1}, \kappa_{2}\right)=1-y_{n}\left(x_{0}, y_{0} ; \kappa_{1}, \kappa_{2}\right) .
\end{aligned}
$$

4. Coordinate translation and parameter reflexion twice [from Eq. (17)],

$$
\begin{aligned}
& x_{n}\left(x_{0}+1 / 2, y_{0} ;-\kappa_{1},-\kappa_{2}\right)=x_{n}\left(x_{0}, y_{0} ; \kappa_{1}, \kappa_{2}\right)+1 / 2, \\
& y_{n}\left(x_{0}+1 / 2, y_{0} ;-\kappa_{1},-\kappa_{2}\right)=y_{n}\left(x_{0}, y_{0} ; \kappa_{1}, \kappa_{2}\right) .
\end{aligned}
$$

5. Coordinate translation and parameter reflexion [from Eq. 18],

$$
\begin{aligned}
& x_{n}\left(x_{0}, y_{0}+1 / 2 ; \kappa_{1},-\kappa_{2}\right)=x_{n}\left(x_{0}, y_{0} ; \kappa_{1}, \kappa_{2}\right)+n \\
& y_{n}\left(x_{0}, y_{0}+1 / 2 ; \kappa_{1},-\kappa_{2}\right)=y_{n}\left(x_{0}, y_{0} ; \kappa_{1}, \kappa_{2}\right)+1 / 2 .
\end{aligned}
$$

6. Coordinate translation and parameter reflexion [from Eq. 19],

$$
\begin{aligned}
x_{n}\left(x_{0}+1 / 2, y_{0}+1 / 2 ;-\kappa_{1}, \kappa_{2}\right)= & x_{n}\left(x_{0}, y_{0} ; \kappa_{1}, \kappa_{2}\right) \\
& +1 / 2+n, \\
y_{n}\left(x_{0}+1 / 2, y_{0}+1 / 2 ;-\kappa_{1}, \kappa_{2}\right)= & y_{n}\left(x_{0}, y_{0} ; \kappa_{1}, \kappa_{2}\right)+1 / 2 .
\end{aligned}
$$

Note that property $P 3$ in (17) implies that if there is an invariant circle above the line $y=0.5$, then there is an invariant circle corresponding to its reflected image bellow $y=0.5$. This same property exists in the standard map, so its invariant circle $\gamma$ has a reflected image (with rotation number $1-\gamma$ ) in the lower half of the cell, and both break up for the same value of the parameter $\kappa_{G}=0.971635406 \ldots$

\section{B. Rotation number symmetries.}

We define the rotation number of an orbit of the map (4) with parameters $\left(\kappa_{1}, \kappa_{2}\right)$,

$$
\omega\left(x_{0}, y_{0} ; \kappa_{1}, \kappa_{2}\right)=\lim _{n \rightarrow \infty} \frac{x_{n}\left(x_{0}, y_{0} ; \kappa_{1}, \kappa_{2}\right)-x_{0}}{n}
$$

whenever the limit exist. 


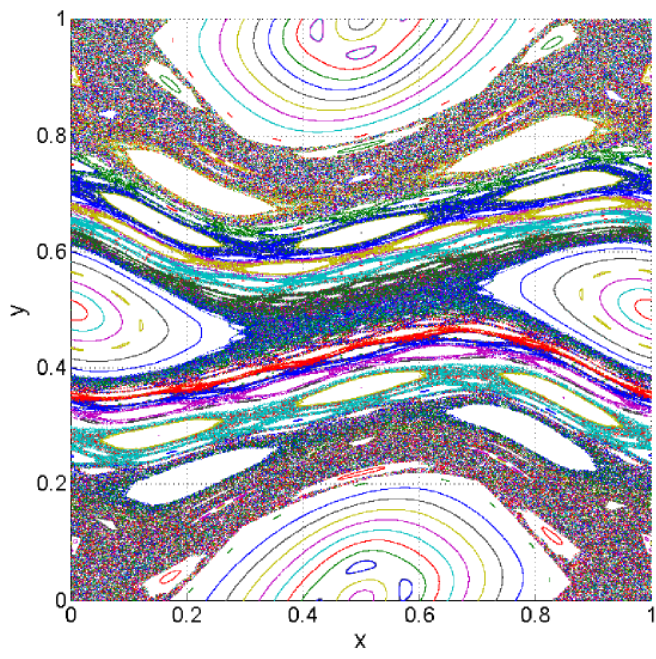

(a)

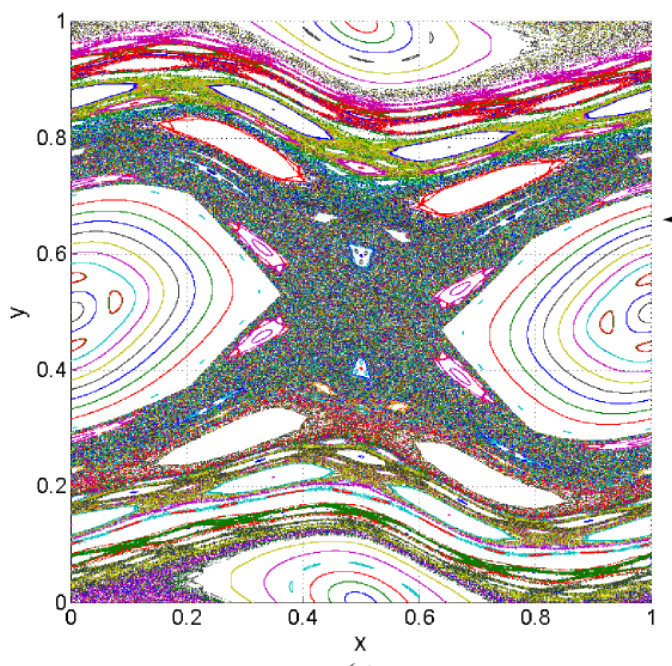

(c)

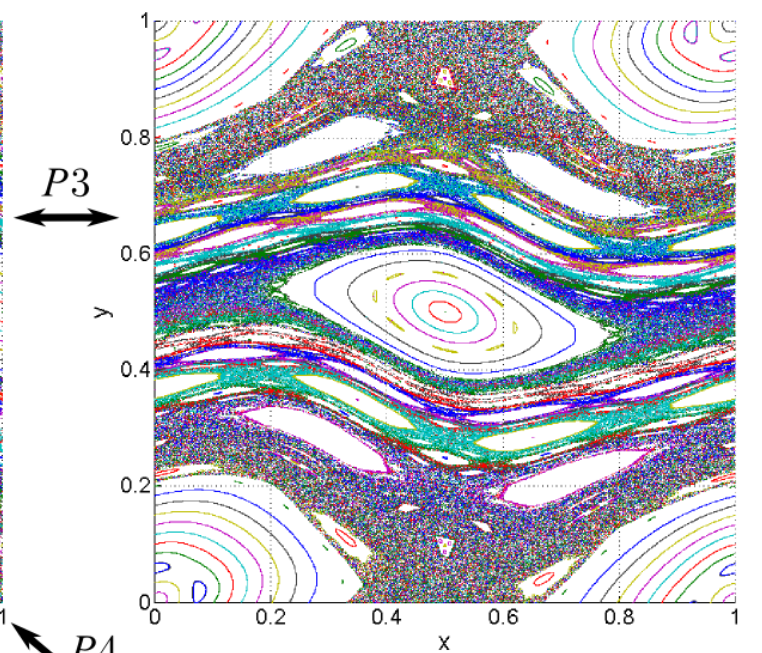

(b)

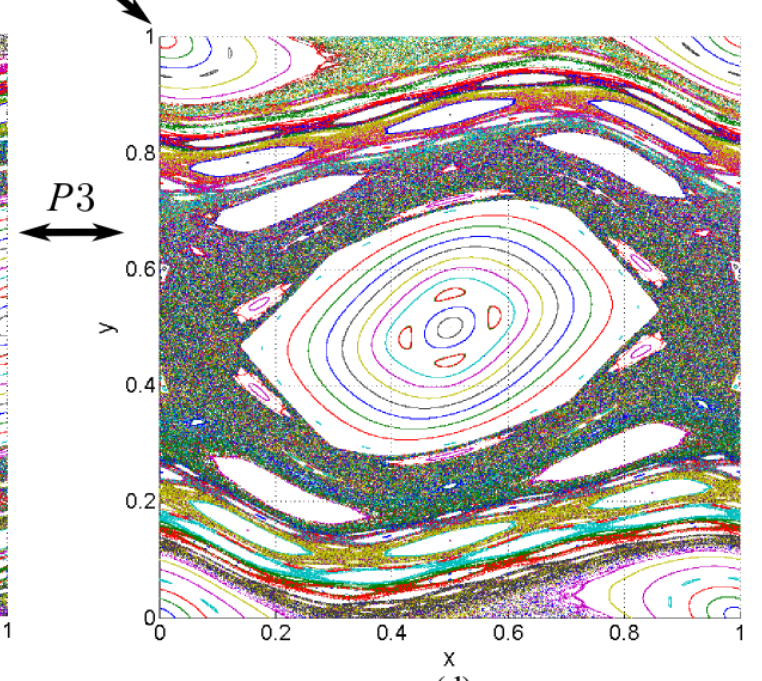

(d)

Figure 2. Phase space of the map (4) for different values of the parameters $\left(\kappa_{1}, \kappa_{2}\right):(\mathrm{a})(0.5,0.7)$ ,(b) $(-0.5,-0.7),(\mathrm{c})(-0.5,0.7)$ and $(\mathrm{d})(0.5,-0.7)$, related by the symmetries $P 3$ and $P 4$, in Eq. (17) and (18) respectively. 
Then from Eqs. (23a), (24a) and (25a), respectively, it follows that

$$
\begin{gathered}
\omega\left(x_{0}+1 / 2, y_{0} ;-\kappa_{1},-\kappa_{2}\right)=\omega\left(x_{0}, y_{0} ; \kappa_{1}, \kappa_{2}\right), \\
\omega\left(x_{0}, y_{0}+1 / 2 ; \kappa_{1},-\kappa_{2}\right)=\omega\left(x_{0}, y_{0} ; \kappa_{1}, \kappa_{2}\right)+1, \\
\omega\left(x_{0}+1 / 2, y_{0}+1 / 2 ;-\kappa_{1}, \kappa_{2}\right)=\omega\left(x_{0}, y_{0} ; \kappa_{1}, \kappa_{2}\right)+1 .
\end{gathered}
$$

Therefore, for each invariant circle passing through $\left(x_{0}, y_{0}\right)$ with rotation number $\omega$ in the NASM with parameters $\left(\kappa_{1}, \kappa_{2}\right)$, there exist up to three other associated invariant circles: one with rotation number $\omega+1$ for $\left(-\kappa_{1}, \kappa_{2}\right)$ passing through $\left(x_{0}+1 / 2, y_{0}+1 / 2\right)$, one also with rotation number $\omega+1$ for $\left(\kappa_{1},-\kappa_{2}\right)$ passing through $\left(x_{0}, y_{0}+1 / 2\right)$, and one with rotation number $\omega$ for $\left(-\kappa_{1},-\kappa_{2}\right)$ passing through $\left(x_{0}+1 / 2, y_{0}\right)$.

In addition, for a given $\left(\kappa_{1}, \kappa_{2}\right)$, from Eq. $(22)$ it can be shown that,

$$
\omega\left(1-x_{0}, 1-y_{0} ; \kappa_{1}, \kappa_{2}\right)=2-\omega\left(x_{0}, y_{0} ; \kappa_{1}, \kappa_{2}\right)
$$

which is a property that also applies to the standard map (6), but with a different shift ${ }^{\mathrm{a}}$.

Finally from Eq. (7) and the definition of rotation number in (26), it follows that,

$$
\omega\left(\mathcal{S}_{\kappa_{2}}\left(\mathbf{z}_{0}\right) ; \kappa_{2}, \kappa_{1}\right)=\omega\left(\mathbf{z}_{0} ; \kappa_{1}, \kappa_{2}\right),
$$

where $\mathbf{z}_{0}=\left(x_{0}, y_{0}\right)^{T}$.

\section{PERIODIC ORBITS.}

The periodic orbits are sets that in many cases offer information that can be used to characterize maps, approximate invariant sets and study linear stability. For these reasons, it is important to study them in the case of the NASM.

The close relationship between $\mathcal{T}_{\kappa_{1} \kappa_{2}}$ and $\mathcal{T}_{\kappa_{2} \kappa_{1}}$ reflects on the fact that if $\mathbf{z}=(x, y)^{T}$ is an $n$-periodic orbit, on the lift of the map ${ }^{\mathrm{b}}$ with rotation number $m / n$ of $\mathcal{T}_{\kappa_{1} \kappa_{2}}$, that is,

$$
\mathcal{T}_{\kappa_{1} \kappa_{2}}^{n}(\mathbf{z})=\mathbf{z}+(m, 0)^{T},
$$

\footnotetext{
a 1 instead of 2 on the right hand side of Eq. (30).

b To the universal cover of $\mathbb{T} \times \mathbb{R}$.
} 
then from Eq. (7) it follows that,

$$
\mathbf{w}=\mathcal{S}_{\kappa_{2}}^{-1} \mathbf{z}
$$

is an $m / n$-periodic orbit of $\mathcal{T}_{\kappa_{2} \kappa_{1}}$, i.e.

$$
\mathcal{T}_{\kappa_{2} \kappa_{1}}^{n}(\mathbf{w})=\mathbf{w}+(m, 0)^{T}
$$

Note that the linear stability properties of $\mathbf{z}$ and $\mathbf{w}$ are the same because the trace of a product of matrices is invariant under the product commutation.

\section{A. Period-one orbits}

There are six primary period-one orbits. By primary we mean that they exist for any values of $\kappa_{1}$ and $\kappa_{2}$. In addition there are bifurcated period-one orbits that exist only for certain values of $\kappa_{1}$ and $\kappa_{2}$. The primary orbits are

$$
\mathcal{P}_{1}=\{(1 / 2,0),(0,0),(0, \pm 1 / 2),(1 / 2, \pm 1 / 2)\}
$$

The stability of these orbits is determined by the residue

$$
R=\frac{1}{4}\left[2-\operatorname{Tr}\left(\nabla \mathcal{T}_{\kappa_{1} \kappa_{2}}\right)\right]
$$

where $\operatorname{Tr}$ denotes the trace, and $\nabla \mathcal{T}_{\kappa_{1} \kappa_{2}}$ is the derivative of the map evaluated at the fixed point. A fixed point $\left(x_{*}, y_{*}\right)$ is stable if and only if $0<R<1$. From this it follows that,

$$
\begin{aligned}
& \text { I. }(0,0) \text { is stable iff } 0<-\kappa_{1}-\kappa_{2}-\frac{\kappa_{1} \kappa_{2}}{2}<2, \\
& \text { II. }\left(\frac{1}{2}, 0\right) \text { is stable iff } 0<\kappa_{1}+\kappa_{2}-\frac{\kappa_{1} \kappa_{2}}{2}<2, \\
& \text { III. }\left(0, \pm \frac{1}{2}\right) \text { is stable iff } 0<\kappa_{2}-\kappa_{1}+\frac{\kappa_{1} \kappa_{2}}{2}<2, \\
& \text { IV. }\left(\frac{1}{2}, \pm \frac{1}{2}\right) \text { is stable iff } 0<\kappa_{1}-\kappa_{2}+\frac{\kappa_{1} \kappa_{2}}{2}<2 .
\end{aligned}
$$

Figure 3 shows the stability regions of the primary period-one fixed points in the $\left(\kappa_{1}, \kappa_{2}\right)$ space, according to (37)-(40). As expected, the results in Fig. 3 are consistent with the symmetries in(15)-(18)).

The secondary period-one orbits $\left(x_{*}, y_{*}\right)$ are solutions to the system,

$$
\begin{aligned}
2 y_{*} & =-\frac{\kappa_{1}}{2 \pi} \sin \left(2 \pi x_{*}\right) \quad \bmod (1), \\
\kappa_{1} \sin \left(2 \pi x_{*}\right) & =-\kappa_{2} \sin \left(2 \pi x_{*}+\frac{\kappa_{1}}{2} \sin \left(2 \pi x_{*}\right)\right) .
\end{aligned}
$$




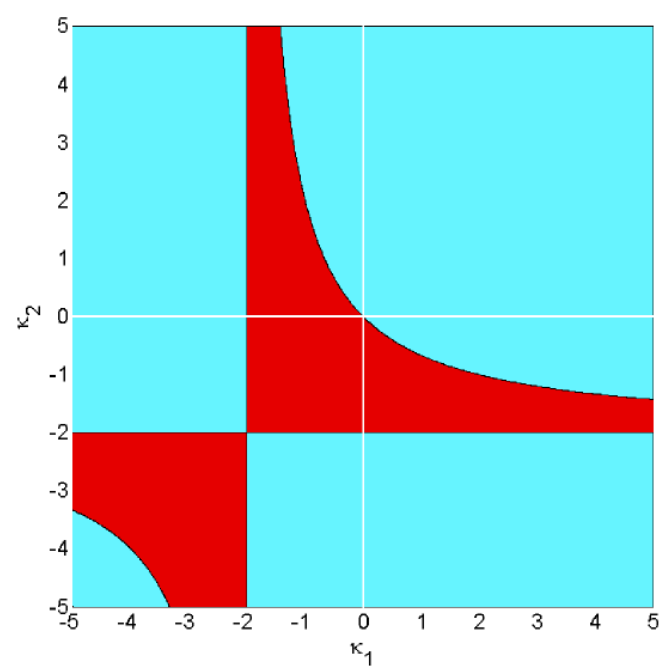

I.

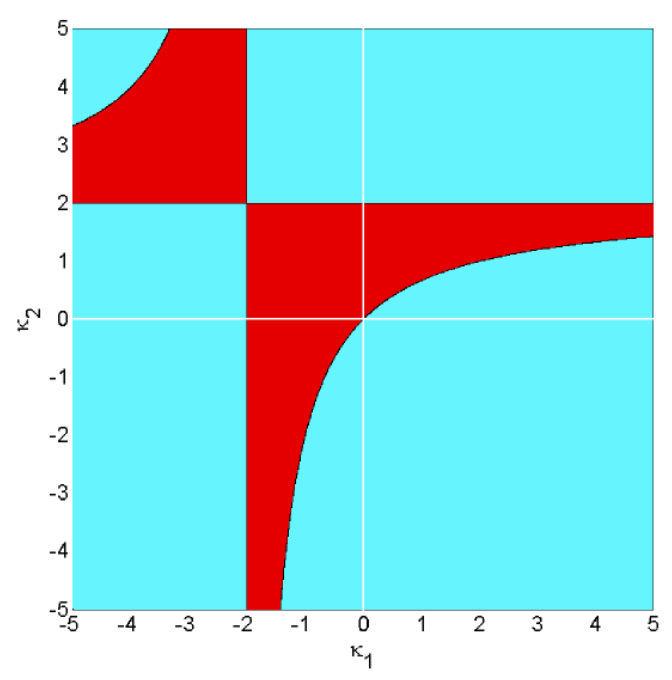

III.

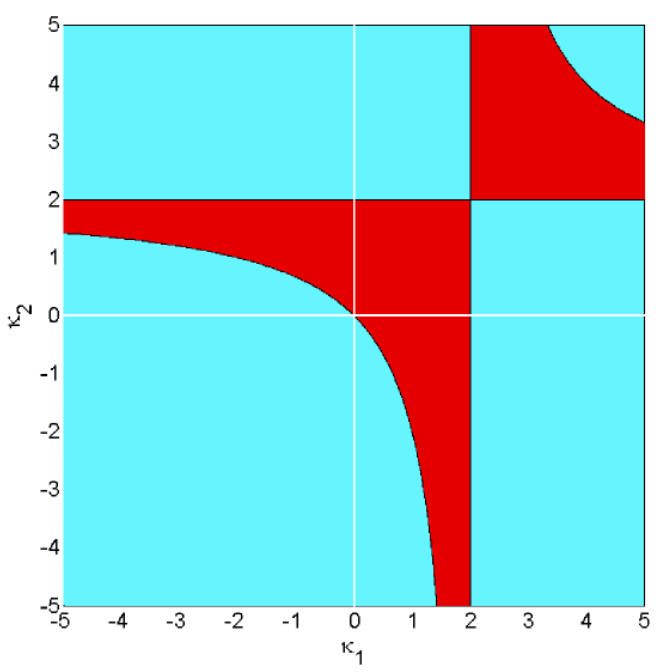

II.

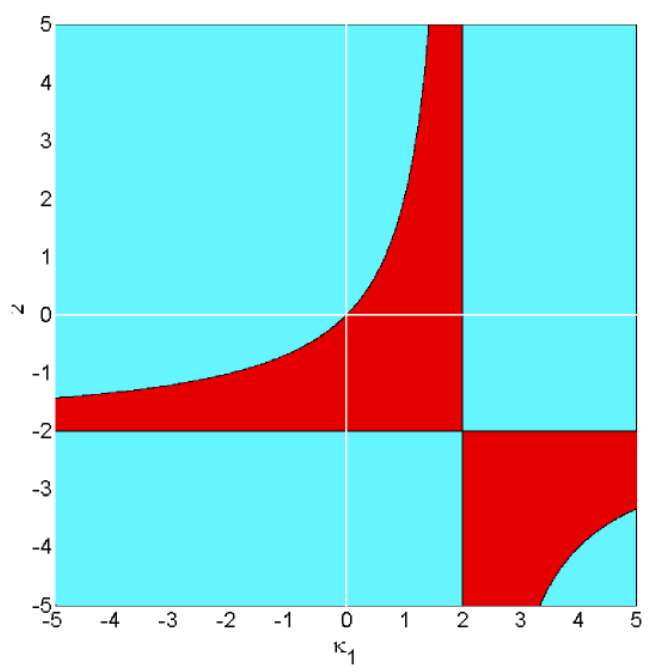

IV.

Figure 3. Stability of primary period-one fixed points. Red regions correspond to stable orbits. As expected, the stability diagrams satisfy the symmetry relations in Eqs. (15)-(18).

A Taylor expansion of (41b) around the elliptic point $(1 / 2,0)$, neglecting fifth order terms, around $\left|z_{*}\right|=\left|2 \pi x_{*}-\pi\right| \ll 1$, allows to estimate $z_{*}$ as

$$
z_{*}^{2}=\frac{6 \kappa_{1}+6 \kappa_{2}+3 \kappa_{1} \kappa_{2}}{\kappa_{1}-\frac{\kappa_{1} \kappa_{2}}{2}+\kappa_{2}\left(1-\frac{\kappa_{1}}{2}\right)^{3}}+O\left(z_{*}^{4}\right) .
$$

Figure 4 shows the region in the positive quadrant of the parameter space where it is possible to find these associated periodic orbits of period 1 around the primary period-one fixed point $\left(\frac{1}{2}, 0\right)$. It should be noted that the limiting curve in the figure coincides with the limiting 
curve in Fig. 3 for the same point. These secondary families of periodic orbits appear after a pitchfork bifurcation of the primary fixed point $\left(\frac{1}{2}, 0\right)$. The value of Greene's residue evaluated at the orbits correspond to the region shaded in red tells us that these orbits are stable.

\section{TRANSPORT BARRIERS: KNOWN LIMIT CASES AND DIRECT COMPUTATION.}

Our goal is to find the critical parameter values of the map (4) for which global transport occurs. We will say that there is global transport when there exists at least one initial condition $\left(x_{0}, y_{0}\right)$ such that the $y$ variable is unbounded. We have observed in (9)-(11) that the map in (4) is symplectic, exact and twist for small values of the parameters. It was established $^{53}$ that for twist maps of the cylinder the only barriers to global transport are invariant circles that are not homotopic to a point. Because of these properties, Moser's Twist Theorem guarantees the existence invariant circles for sufficiently small values of the parameters $\kappa_{i}$ in a neighborhood in parameter space of the origin which corresponds to the integrable case. the map is integrable. Then it is reasonable to compute the critical boundaries $(C B)$ in the parameter space for the onset of global transport $\left(C B_{g t}\right)$ and for the destruction of invariant circles with a fixed rotation number, $\omega,\left(C B_{\omega}\right)$. By $C B$ we mean the boundary of the open region $\mathcal{R}$ in the parameter space (assumed to be simply connected) such that for $\left(\kappa_{1}, \kappa_{2}\right)$ values in $\mathcal{R}$ there is not global transport in the case of $\left(C B_{g c}\right)$ or a given invariant circle with rotation number $\omega$ exists in the case of $\left(C B_{\omega}\right)$.

We propose three different approaches to find these transport barriers: analytical reductions of the map for particular values of the parameters, direct numerical iteration for a range of values of the parameters which gives upper bounds, and a continuation method over invariant circles with an a priori chosen rotation number in the parameter space which gives lower bounds to global transport.

\section{A. Known limit cases.}

In the standard map, the term critical parameter value, $\kappa_{c}$, is established in the literature ${ }^{54}$ as the value for with any further increment $|\kappa|>\left|\kappa_{c}\right|$ there are no invariant curves. We call 


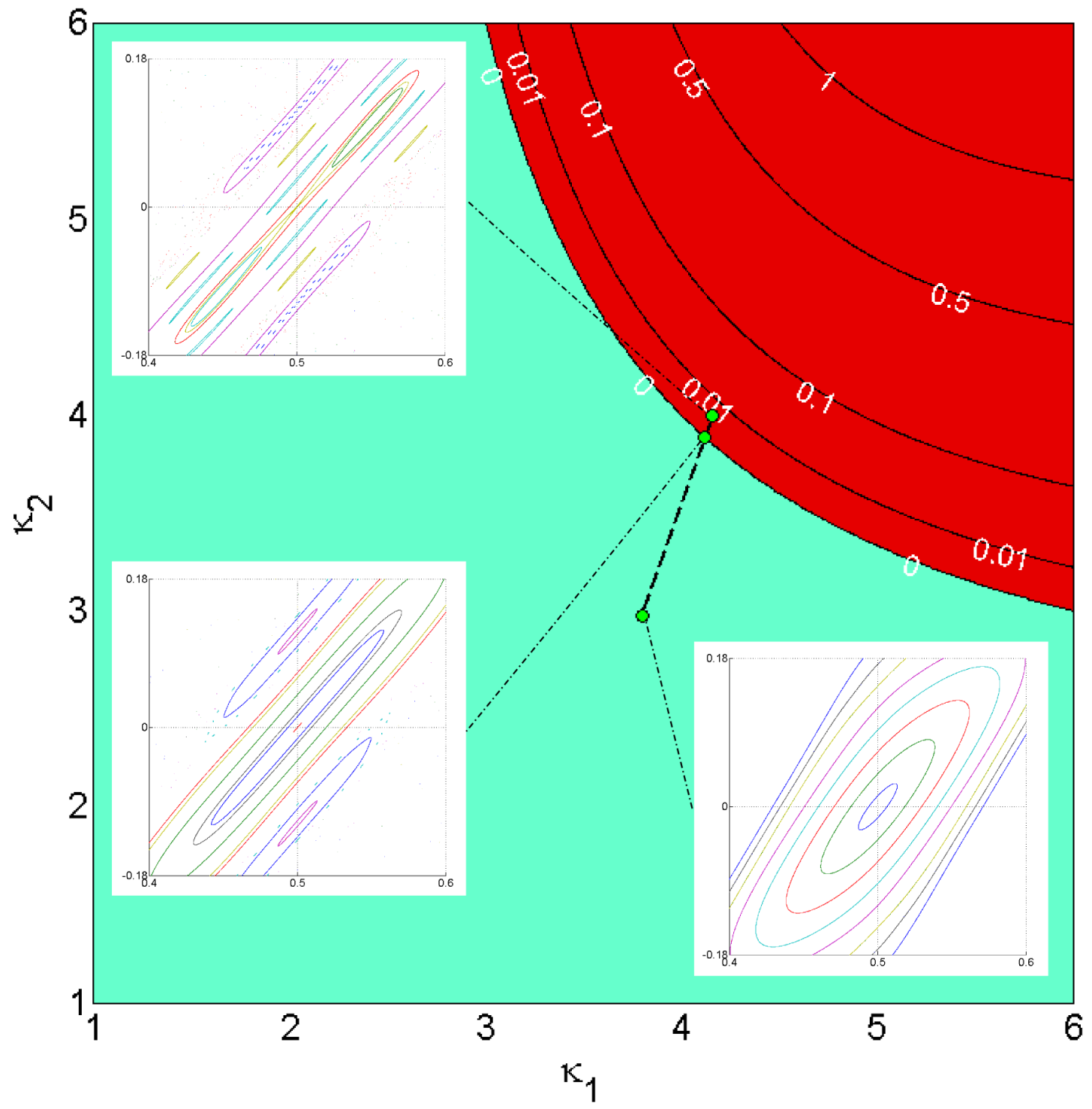

Figure 4. Region of existence of the secondary period 1 periodic orbits around the elliptic point $\left(\frac{1}{2}, 0\right)$ in the positive quadrant of the parameter space. The light green shaded region corresponds to values of the parameters where the secondary periodic orbits do not exist. The figure displays in red the increasing values of the absolute error of the approximate value of $z_{*}$ from (42), when substituted into (41b). The red colored region also coincides with values of the Greene's residue, Eq. (36), for when the orbits are stable.

critical invariant curve to such invariant curve that exist for $\kappa=\kappa_{c}$ and cease to exist after the critical value. It is a known conjecture ${ }^{54}$ that for the standard map, the rotation number of the critical invariant curve is equal to the golden mean $\gamma=\frac{\sqrt{5}-1}{2}$. 
We present some particular cases of map (4).

1. $\left(\kappa_{1}, \kappa_{2}\right)=\left(\kappa_{1}, 0\right), \kappa_{1} \geq 0$.

In this case the map (4) reduces to,

$$
\begin{aligned}
& x_{n+1}=x_{n}+2 y_{n}+\frac{2 \kappa_{1}}{2 \pi} \sin \left(2 \pi x_{n}\right) \\
& y_{n+1}=y_{n}+\frac{\kappa_{1}}{2 \pi} \sin \left(2 \pi x_{n}\right)
\end{aligned}
$$

which upon the change of coordinates: $\{X=x, Y=2 y\}$, becomes the standard map (6) with $\epsilon=2 \kappa_{1}$. And it is well known in this case that the critical invariant circle has rotation number equal to the golden mean $\gamma$ and breaks at the critical value $\kappa_{G} \approx 0.971635406^{35,54}$. This implies that the critical invariant circle for the case $\left(\kappa_{1}, \kappa_{2}\right)=\left(\kappa_{1}, 0\right)$ has rotation number $\omega_{c}=\gamma$ and breaks for $\kappa_{1 c}=\kappa_{G} / 2$. Applying Eq. (30) to the standard map reduction and the NASM, it follows that there are also three more critical invariant circles with rotation numbers: $1-\gamma, 2-\gamma$ and $\gamma+1$.

2. $\left(\kappa_{1}, \kappa_{2}\right)=\left(0, \kappa_{2}\right), \kappa_{2} \geq 0$.

In this case the map (4) reduces to,

$$
\begin{aligned}
& x_{n+1}=x_{n}+2 y_{n}+\frac{\kappa_{2}}{2 \pi} \sin \left(2 \pi\left(x_{n}+y_{n}\right)\right), \\
& y_{n+1}=y_{n}+\frac{\kappa_{2}}{2 \pi} \sin \left(2 \pi\left(x_{n}+y_{n}\right)\right) .
\end{aligned}
$$

As before, with the change of coordinates: $\{X=x+y, Y=2 y\}$, the map reduces to the standard map (6) with perturbation parameter $\epsilon=2 \kappa_{2}$. Therefore the critical invariant circle has rotation number $\omega_{c}=\gamma$ and breaks for $\kappa_{2 c}=\kappa_{G} / 2$. From Eq. (30), there are three more critical invariant circles: $1-\gamma, 2-\gamma$ and $\gamma+1$. This case can also be considered a consequence of the Eq. (31) applied to the previous case.

3. $\left(\kappa_{1}, \kappa_{2}\right)=(\kappa, \kappa), \kappa \geq 0$.

In this case the map reduces to the standard map iterated twice, which that the critical invariant circle of map (4), has rotation number $\omega_{c}=2 \gamma$ and breaks at $\kappa_{c}=\kappa_{G}$. By the symmetry (30), there exists also the invariant circle with rotation number: $\omega_{c_{2}}=2-2 \gamma$. 
Furthermore, applying the results of section III.B we can give the rotation numbers of the critical circles in the other quadrants. For example, for $(-\kappa, \kappa)$, the critical invariant circles are: $\omega_{c_{1}}=2 \gamma-1$ and $\omega_{c_{2}}=3-2 \gamma$ for $\kappa_{c}=\epsilon_{c}$.

It should be noted that the change of variables used in the first two cases are homotopic to the identity, so the barriers in the cylinder for the standard map reductions are barriers for the NASM as well.

\section{B. Direct computation.}

We formally define a barrier to global transport as an invariant circle not homotopic to a point, which geometrically are circles that go around the cylinder $\mathbb{T} \times \mathbb{R}$. An invariant circle of this kind, when it exists, always divides the phase space in two unbounded invariant regions, due to its invariance and the continuity of the map. This method is basically a modified version of the crossing method introduced by Chirikov ${ }^{55}$.

The numerical computation of the threshold to global transport is based on the following criteria:

Proposition 1. (Global transport criterion)

If for a set value of the parameters $\left(\kappa_{1}, \kappa_{2}\right)$, there is an initial condition $\left(x_{0}, y_{0}\right)$ with $y_{0} \in$ $(0,1)$, for which $\left|y_{n}-y_{0}\right|>2$ for some $n$ and all the invariant circles of the map have an amplitude $\mathcal{A}<1$, then the map has global transport.

Proof. Lets assume that for the map with $\left(\kappa_{1}, \kappa_{2}\right)$, there exists an initial condition $\left(x_{0}, y_{0}\right)$ such that $\left|y_{n}-y_{0}\right|>2$ for a certain $n$ but there still exists an invariant circle fully contained in the cell $[0,1] \times(Q, Q+1)$ for $Q \in \mathbb{R}$. By the orbit symmetry property in Eq. (21), using $r=0$ and $s=-\lfloor Q\rfloor:=-\max \{m \in \mathbb{Z} \mid m \leq Q\}$, there exists a copy of the invariant circle in $[0,1] \times[0,2]$. Then, either the invariant circle or one of its copies $(s=-\lfloor Q\rfloor \pm 1)$ lies between $y_{0}$ and $y_{n}$. Which is a contradiction because $y_{0}$ and $y_{n}$ must be in the same connected component of the cylinder.

Numerical evidence, see e.g. Fig. 2(a), shows that at least for parameter values $\left|\kappa_{i}\right|<1$, the hypothesis, $\mathcal{A}<1$, holds. The hypothesis is also obeyed because the invariant stable

${ }^{\mathrm{c}}$ Where $\mathcal{A}$ is the height difference between the highest and lowest point of the invariant circle. 
and unstable manifolds of the hyperbolic fixed point around $(0,0)$ obstruct the path of the invariant curve, and so do its integer translates in the $y$-direction.

We performed several series of $N$-iterations of the map (4) on $M$ initial conditions taken uniformly distributed in the rectangle $[0,1] \times[0,0.3]$ to determine, using the proposed transport criteria (Proposition 1) when the map displayed global transport for a wide range of the parameters $\left(\kappa_{1}, \kappa_{2}\right)$ inside the twist region. Different number of initial conditions, $M$, were used, finding $M=10^{4}$ to be a reasonable balance between the computing capabilities and the consistency of the results. The procedure was repeated for an increasing number of iterations $N$. The convergence of the method is shown in Figure 7. Figures 5 and 6 show the critical boundary for the global transport $\left(C B_{g t}\right)$ found with these calculations, i.e. the locus of points in parameter space for which no critical invariant curves were detected.

In all the cases studied, the $C B_{g t}$ in the upper half plane of the parameter space were symmetric (up to machine precision) on the right (left) quadrant with respect to the line $\kappa_{2}=\kappa_{1}\left(\kappa_{2}=-\kappa_{1}\right)$, Fig. 6 .

The slope of the adjusted line to the convergence data obtained with the direct method for a variable number of iterations $N$, Fig. 7 , suggests that the convergence of the method is algebraic: $\left(\kappa_{N}-\kappa_{G}\right) \propto N^{-1 / \eta}$, with $\eta=2.681$ (and $\eta=3.322$ for large $N$ ), which is close to the value, $\eta=3.012$, reported in literature ${ }^{35}$ for transport in the standard map.

\section{TRANSPORT BARRIERS: CONTINUATION METHOD.}

In this section we describe the numerical implementation of the computation and numerical continuation of invariant circles of the map in Eq. (4). We will omit most of the technical details and only discuss the tools that we will use. For further mathematical details (e.g. function spaces, geometric preliminaries, and Diophantine properties) the reader is referred to Ref. 56-59.

Definition 1. We say that the irrational number $\omega$ is Diophantine if for a given $\tau$ there is a constant $\nu$ such that,

$$
|\omega \cdot q-p| \geq \nu|q|^{-\tau}, p \in \mathbb{Z}, q \in \mathbb{Z} \backslash\{0\} .
$$

We will denote the set of all numbers satisfying Definition 1 by $\mathcal{D}(\nu, \tau)$. 


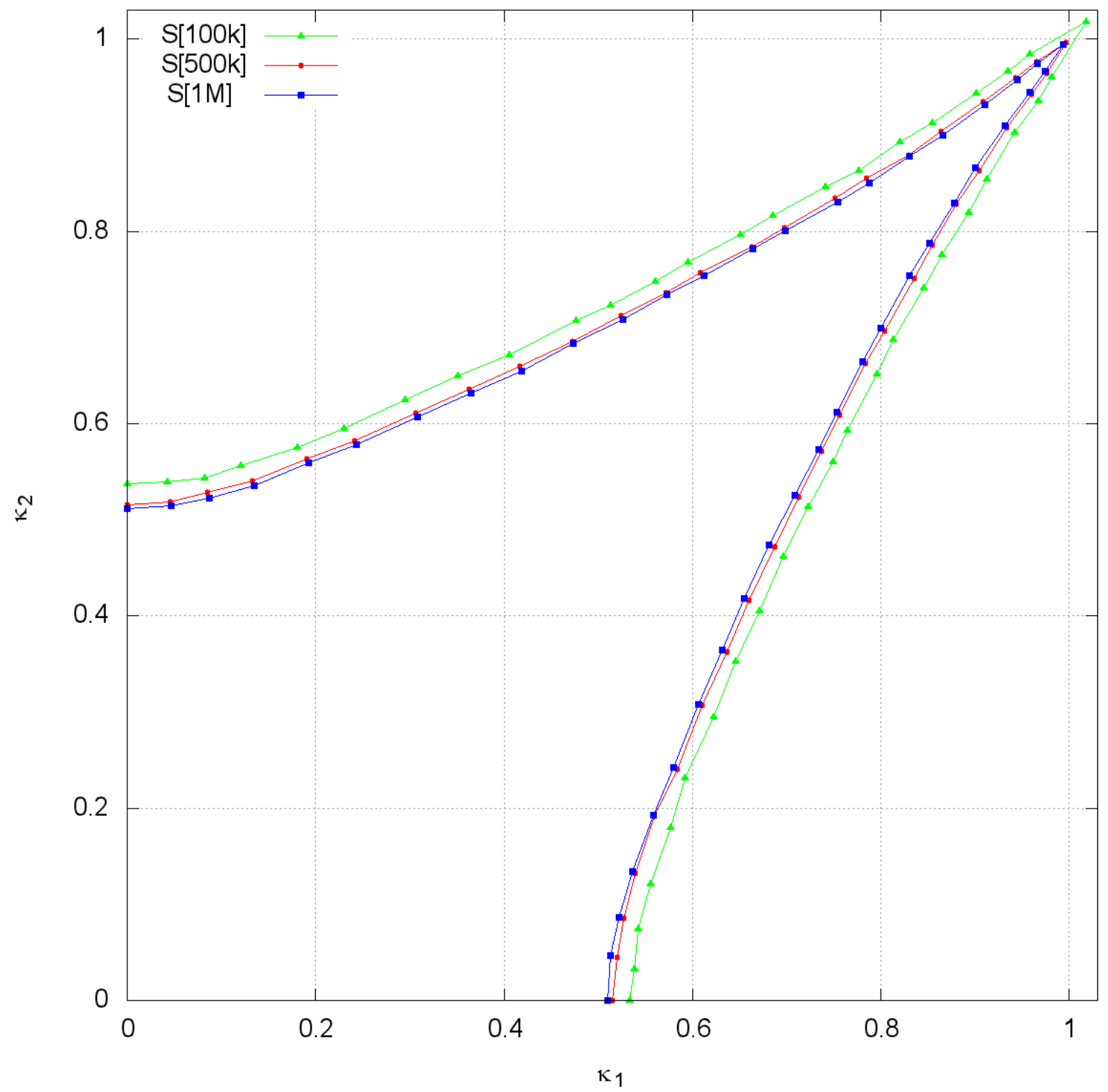

Figure 5. Critical boundary for global transport $\left(C B_{g t}\right)$ in the non-autonomous standard map in Eq. (4), in the positive quadrant. The area outside the "horn" corresponds to parameter values for which there is global transport, in the sense that at least one of $10^{4}$ initial condition uniformly distributed on the rectangle $[0,1] \times[0,0.3]$ exhibited a displacement with: $y>0.3+2$ or $y<-2$ after $10^{5}$ (green triangles), $5 \times 10^{5}$ (red circles) and $10^{6}$ (blue squares) iterations of the map.

Then, we look for invariant circles of $\mathcal{T}_{\kappa_{1} \kappa_{2}}$ on which the dynamics is conjugated to a rigid rotation by a fixed Diophantine rotation number $\omega$.

The method we use is best understood in the constructive proof of the KAM theorem in Ref. 58, which relies among other things in a Newton iteration in the spirit of Nash-Moser 


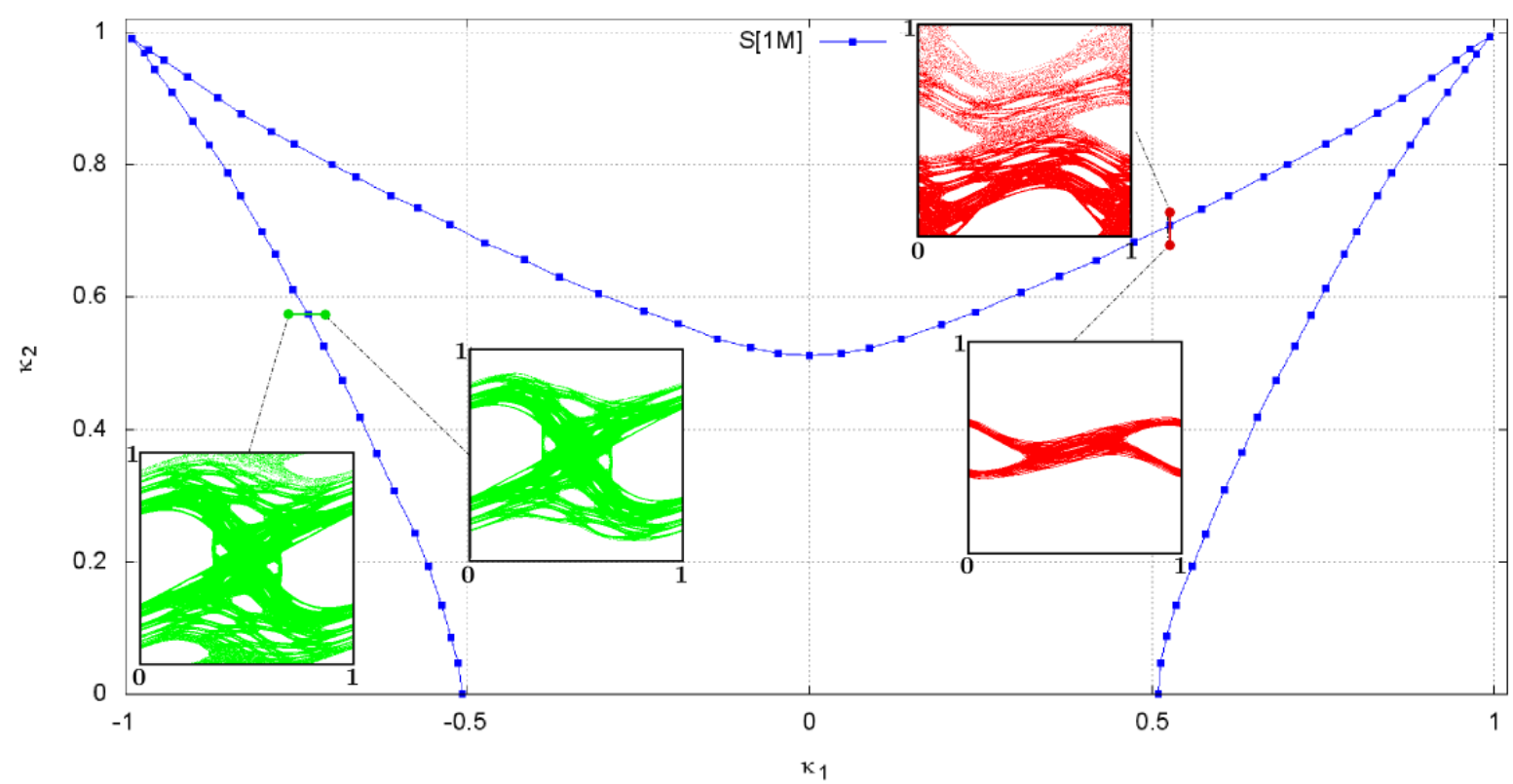

Figure 6. Critical boundary for global transport $\left(C B_{g t}\right)$ in the non-autonomous standard map in Eq. (4) in the upper half plane of parameter space, obtained by the direct method with $M=10^{4}$ and $N=10^{6}$. As in Figure 5, the area outside the "horns" corresponds to global transport, for the same conditions as in Fig. 5. The insets are images of the cell $[0,1] \times[0,1]$ of the phase space $(x, y)$ obtained from the iteration $\left(N=3 \times 10^{6}\right)$ of the NASM for the initial condition $(0.5,0.44)$ for parameter values close the computed boundary: $(0.526,0.69),(0.526,0.73),(-0.69,0.525)$ and $(-0.73,0.525)$.

theory, see Ref. 60. For us, the main advantage of using the constructive proof is that it leads to a very efficient numerical algorithm. We will make use of the Nash-Moser techniques to produce algorithms that allow us to continue smooth functions $K: \mathbb{T} \rightarrow \mathbb{T} \times \mathbb{R}$ which satisfy the invariance equation,

$$
F \circ K(\theta)=K(\theta+\omega)
$$

where $F: \mathbb{T} \times \mathbb{R} \rightarrow \mathbb{T} \times \mathbb{R}$ if the given twist map and $\omega \in \mathcal{D}(\nu, \tau)$. Starting from the integrable case of the map $F$ and moving the parameter as close to the breakdown of analyticity of the invariant circles as possible. We use the criterion of breakdown in Ref. 57, namely when we are close to the breakdown of analyticity the derivatives of the solution $K$ start to blow up at points of $K(\mathbb{T})$. 


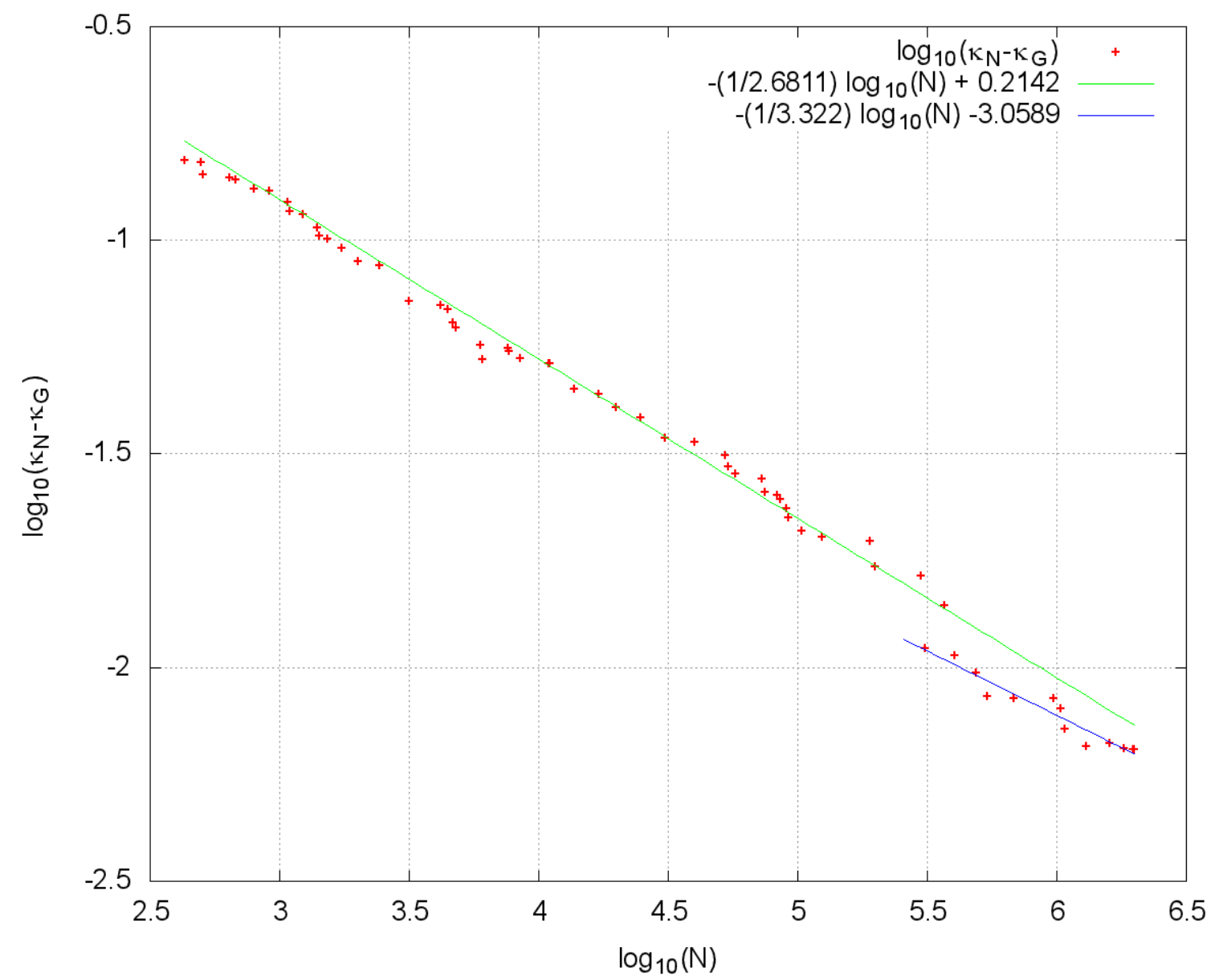

Figure 7. Convergence of the critical parameter value $\kappa_{c}$ as function of the number of iterates $N$, observed using the direct method at the cusp of the horn, along the diagonal $\left(\kappa_{1}, \kappa_{2}\right)=(\kappa, \kappa)$. To better display the results, the plotted quantity on the vertical axis is $\kappa_{N}-\kappa_{G}$.

Continuation methods like the one presented here have already been used in several contexts. See for instance, Ref. 47 and 61, for models in statistical mechanics, Ref. 62-64 for examples in symplectic maps, Ref. 48 and 65 for conformally symplectic models, and Ref. 66 for volume preserving maps.

The main idea of the method is to start form an approximate solution of the invariance equation applied to $\mathcal{T}_{\kappa_{1} \kappa_{2}}$. We will say that the solution $K_{0}$ is approximately invariant if

$$
e_{0}(\theta)=\mathcal{T}_{\kappa_{1} \kappa_{2}} \circ K_{0}(\theta)-K_{0}(\theta+\omega)
$$

and $\left\|e_{0}\right\|$ is a small function with respect to the norm $\|\cdot\|$ of the Banach space of smooth functions.

We produce a "better" approximate solution (a solution that approximates the invariance equation (45) with a smaller error), by adding a periodic function $\Delta: \mathbb{T} \rightarrow \mathbb{R} \times \mathbb{T}$ so that 
$K_{1}(\theta)=K_{0}(\theta)+\Delta(\theta)$ has an error

$$
e_{1}(\theta)=\mathcal{T}_{\kappa_{1} \kappa_{2}} \circ K_{1}(\theta)-K_{1}(\theta+\omega)
$$

with $\left\|e_{1}\right\| \approx\left\|e_{0}\right\|^{2}$.

Indeed, according to Nash-Moser theory, adding an appropriate correction $\Delta$ could provide an error satisfying the quadratic property above. The correction $\Delta$ we could use, would solve the Newton step equation,

$$
D \mathcal{T}_{\kappa_{1} \kappa_{2}}\left(K_{0}(\theta)\right) \Delta(\theta)-\Delta(\theta+\omega)=-e_{0}(\theta)
$$

It is easy to check that if we were able to solve for $\Delta$ form equation (46), then the norm of the new error, $\left\|e_{1}\right\|$ will be of order $\left\|e_{0}\right\|^{2}$.

One can try to solve numerically the Newton equation in (46) for $\Delta$, but the most efficient methods would require $O\left(n^{3}\right)$ operations, where $n$ is the number of points that one uses to represent the invariant circle. The alternative that we will follow is to reduce the Newton step equation (46) by introducing a symplectic change of coordinates around the approximate solutions. The implementation will yield methods that require $O(n \log n)$ operations.

The change of coordinates is around an approximate solutions $K_{0}$ is given by a 2 by 2 matrix composed of two column vectors. The first 2 by 1 column is the vector $D K_{0}(\theta)$ representing the tangent bundle to the approximate solution with base at every point $K(\theta)$. The second column vector is a symplectic conjugate bundle which in our case of one dimensional circles reduces to a vector orthogonal to the tangent bundle, namely $J^{-1} D K_{0}(\theta) N_{0}(\theta)$, where $J$ is the matrix representation of the symplectic form $\Omega$ and

$$
N_{0}(\theta):=\left[D K_{0}(\theta)^{T} D K_{0}(\theta)\right]^{-1} .
$$

So the matrix can be written as follows,

$$
M_{0}(\theta)=\left[D K_{0}(\theta) \mid J^{-1} D K_{0}(\theta) N_{0}(\theta)\right] .
$$

This change of coordinates is symplectic and transforms approximately the matrix $D \mathcal{T}_{\kappa_{1} \kappa_{2}}\left(K_{0}(\theta)\right)$ into an upper triangular matrix with ones along the diagonal, namely,

$$
D \mathcal{T}_{\kappa_{1} \kappa_{2}}\left(K_{0}(\theta)\right) M_{0}(\theta)=M_{0}(\theta+\omega)\left(\begin{array}{cc}
1 & S_{0}(\theta) \\
0 & 1
\end{array}\right)
$$


where

$$
S_{0}(\theta)=N_{0}(\theta+\omega) D K_{0}^{T}(\theta+\omega) D \mathcal{T}_{\kappa_{1} \kappa_{2}}\left(K_{0}(\theta)\right) D K_{0}(\theta) N_{0}(\theta)
$$

The function $S_{0}(\theta)$ is related to the local twist condition on the invariant circle $K_{0}$. We apply the change of coordinates to the Newton step, $\Delta(\theta)=M_{0}(\theta) W(\theta)$, to reduce approximately the Newton step equation. Indeed, the new Newton step now has to solve the equation,

$$
\begin{aligned}
\left(\begin{array}{cc}
1 & S_{0}(\theta) \\
0 & 1
\end{array}\right)\left(\begin{array}{l}
W_{1}(\theta) \\
W_{2}(\theta)
\end{array}\right) & -\left(\begin{array}{l}
W_{1}(\theta+\omega) \\
W_{2}(\theta+\omega)
\end{array}\right)= \\
& -M_{0}^{-1}(\theta+\omega) e_{0}(\theta) .
\end{aligned}
$$

Now, if we split the equation (49) into components we obtain two cohomological equations that we need to solve, namely

$$
W_{2}(\theta)-W_{2}(\theta+\omega)=-\left[M_{0}^{-1}(\theta+\omega) e_{0}(\theta)\right]_{2}
$$

and

$$
W_{1}(\theta)-W_{1}(\theta+\omega)=-\left[M_{0}^{-1}(\theta+\omega) e_{0}(\theta)\right]_{1}-S(\theta) W_{2}(\theta)
$$

From equation (11), we know that $\mathcal{T}_{\kappa_{1} \kappa_{2}}$ is exact. Then it can be shown (Lemma 9 from Ref. 58) that

$$
\int_{\mathbb{T}}\left[M_{0}^{-1}(\theta+\omega) e_{0}(\theta)\right]_{2} d \theta=O\left(\left\|e_{0}\right\|^{2}\right)
$$

Therefore, we are able to solve for $\Delta$ form (49) by following the algorithm described below.

\section{Algorithm 1.}

1) Let $e_{0}(\theta)=\mathcal{T}_{\kappa_{1} \kappa_{2}} \circ K_{0}(\theta)-K_{0}(\theta+\omega)$

3) Compute the matrix $M(\theta)$ from equation (47).

4) Solve for $W_{2}(\theta)$ from (50).

5) Choose the average $\int_{\mathbb{T}} W_{2}(\theta) d \theta$ so that $-\left[M_{0}^{-1}(\theta+\omega) e_{0}(\theta)\right]_{1}-S_{0}(\theta) W_{2}(\theta)$ has an average close to zero.

6) Solve for $W_{1}(\theta)$ from (51).

7) Compute the step $\Delta$,

$$
\Delta(\theta)=M_{0}(\theta) W(\theta)
$$


6) Obtain the new parameterization $K_{1}$,

$$
K_{1}(\theta)=K_{0}(\theta)+\Delta(\theta)
$$

7) Set $K_{0}(\theta)=K_{1}(\theta)$ and go to step 1$)$.

Remark. We note that in order to implement the computations in step 5, one has to make sure that the twist condition is satisfied. Verifying the twist condition might be a drawback when we apply the algorithm to maps that do not have a uniform twist condition. However in our case, we only required to perform the continuation in parameter regions where the maps satisfied the twist condition uniformly.

Remark. One can verify that all the operations required to implement algorithm 1, are either diagonal in Fourier space of in real space. To transform from real space to Fourier space one can use a Fast Fourier Transform (FFT), which is the most expensive operation in the Algorithm 1 in terms of arithmetic operations. Therefore, the cost of implementing Algorithm 1 is $O(n \log n)$ operations with $O(n)$ storage requirements, where $n$ is the number of points used to represent the circle.

In the following section we emphasize that there are useful relations between the parameterization method exposed here and the symmetries that were introduced in Section III.

\section{A. Symmetries of the parameterization of an invariant circle}

In this Section we present some of the symmetries discussed in Section III from the point of view of the parameterization method. The goal is to rewrite the symmetries in terms of compositions of functions. In this way, we can use this composition formulation to rewrite the invariance equation that a parameterization of a circle with a certain symmetry should satisfy. This way we know that if a map has an invariant circle, then we can use that parameterization function, $K$, and transform it using the composition operators with respect to a given symmetry. In this way, we construct a new parameterization that satisfies the invariance equation of a new map with the given symmetry. We start by verifying that the invariant circles of the map $\mathcal{T}_{\kappa_{1}, \kappa_{2}}$ are expected to exist and to have the rotation numbers found in Section VA. 
First, we consider that the map $F$ has an invariant circle with rotation number $\omega$, whenever Eq. (45) is satisfied. For instance, if $S_{\kappa}$ in Eq. (6), has an invariant circle of rotation number $\omega$, with graph $K$, then $\mathcal{T}_{\kappa \kappa}$ will have an invariant torus with rotation number $2 \omega$ as described above. Since,

$$
S_{\kappa} \circ K(\theta)=K(\theta+\omega)
$$

and

$$
S_{\kappa} \circ K(\theta+\omega)=K(\theta+2 \omega)
$$

then

$$
S_{\kappa} \circ S_{\kappa} \circ K(\theta)=S_{\kappa} \circ K(\theta+\omega)=K(\theta+2 \omega) .
$$

We also notice that if we define the following function for $\phi \in \mathbb{R}$,

$$
R_{\phi}(x, y)=(x+\phi \bmod 1, y)
$$

then the standard map (4) satisfies that,

$$
S_{\epsilon} \circ R_{-\frac{1}{2}}=R_{-\frac{1}{2}} \circ S_{-\epsilon}
$$

for any $\epsilon$. We have one more symmetry of the parameterization that will be important. To see this, we write the parameterization of an invariant circle of a symplectic map of the cylinder, we write the components of the parameterization $K$ as follows. Let $u(\theta)$ be a 1-periodic function of $\mathbb{T}$, then

$$
K(\theta)=\left(\begin{array}{c}
\theta+u(\theta) \\
\omega+u(\theta)-u(\theta-\omega)
\end{array}\right) .
$$

It is clear from (54) and the periodicity of $u$, that

$$
R_{-1} \circ K(\theta)=K(\theta-1)
$$

In particular, if $K$ is the parameterization of an invariant circle of $S_{\kappa} \circ R_{-\frac{1}{2}}$ then by the property (52) of the square of a map above, we have that

$$
S_{\kappa} \circ R_{-\frac{1}{2}} \circ S_{\kappa} \circ R_{-\frac{1}{2}} \circ K(\theta)=K(\theta+2 \omega) .
$$

So by the symmetry property in (53) of the Standard map together with the symmetry (55) of the invariant circle, we have that

$$
\begin{aligned}
S_{\kappa} \circ S_{-\kappa} \circ R_{-1} \circ & K(\theta)=K(\theta+2 \omega) \\
& \Longrightarrow S_{\kappa} \circ S_{-\kappa} \circ K(\theta-1)=K(\theta+2 \omega)
\end{aligned}
$$


This is equivalent to saying that $K$ is an invariant circle of $S_{\kappa} \circ S_{-\kappa}$ with rotation number $2 \omega+1$, where $K$ is the invariant circle of $S_{\kappa} \circ R_{-\frac{1}{2}}$ with rotation number $\omega$, which is the same as the symmetry in Eq. (28).

We verify the properties formulated in Section $\mathrm{V}$ that state that the maps $\mathcal{T}_{\kappa_{1} 0}$ and $\mathcal{T}_{0 \kappa_{2}}$ can be rescaled to standard maps with twice the perturbation parameter.

If we define the transformation

$$
P_{\xi}(x, y)=(x \bmod 1, \xi y)
$$

as simple computation tells us that

$$
\mathcal{T}_{\kappa_{1} 0}=S_{0} \circ S_{\kappa_{1}}=P_{1 / 2} \circ S_{2 \kappa_{1}} \circ P_{2}
$$

So if $S_{2 \kappa_{1}}$ has an invariant circle, then the invariance equation is

$$
S_{2 \kappa_{1}} \circ K(\theta)=K(\theta+\omega) .
$$

We immediately know that $P_{1 / 2} \circ K(\theta)$ is an invariant circle for $\mathcal{T}_{\kappa_{1} 0}$. Namely,

$$
P_{2} \circ S_{0} \circ S_{\kappa_{1}} \circ P_{1 / 2} \circ K(\theta)=K(\theta+\omega),
$$

is equivalent to

$$
\mathcal{T}_{\kappa_{1} 0} \circ P_{1 / 2} \circ K(\theta)=P_{1 / 2} \circ K(\theta+\omega) .
$$

The case of the map $\mathcal{T}_{0 \kappa_{1}}$ is obtained for the above since when we use (7), we have that $\mathcal{T}_{0 \kappa_{1}}=S_{0}^{-1} \circ \mathcal{T}_{\kappa_{1} 0} \circ S_{0}$. Then, it is clear that the circle $S_{0}^{-1} \circ P_{1 / 2} \circ K$ is invariant for $\mathcal{T}_{0 \kappa_{1}}$. In fact, from Eq. 7 it is easy to see that if $\mathcal{T}_{\kappa_{1} \kappa_{2}}$ has an invariant circle with rotation number $\omega$, then $\mathcal{T}_{\kappa_{2} \kappa_{1}}$ has an invariant circle with the same rotation number. This result was also stated in Eq. (31).

\section{B. Results from the parameterization method}

The parameterization method was applied using the rotation numbers obtained from

section $\mathrm{V}: \gamma, \gamma+1,2 \gamma$ and $2 \gamma+1$ and also with $\frac{5 \gamma+6}{4 \gamma+5}$ and $\frac{\gamma+1}{4 \gamma+5}$ which were found heuristically. The method yielded in all the cases as critical boundaries between the existence and breaking of a given invariant circles with rotation number $\omega,\left(C B_{\omega}\right)$, a two horn-shaped asymmetrical curve in the upper plane of the parameter space $\left(\kappa_{1}, \kappa_{2}\right)$. The points obtained in the $C B_{\omega}$ 
correspond to the blow-up of the Sobolev norms of the parameterization function $K$. The critical boundaries are displayed in Figure 8 with the addition of the a curve of the first method to contrast the results. All the critical boundaries agree with the results from section $\mathrm{V}$ and are contained in the tightest curve from previous subsection. Notice that the $C B$ corresponding to $\gamma$ is related to the one of $\gamma+1$ and the $C B$ of $2 \gamma$ is related to the one of $2 \gamma+1$ by a reflection with respect to the $\kappa_{2}$-axis.

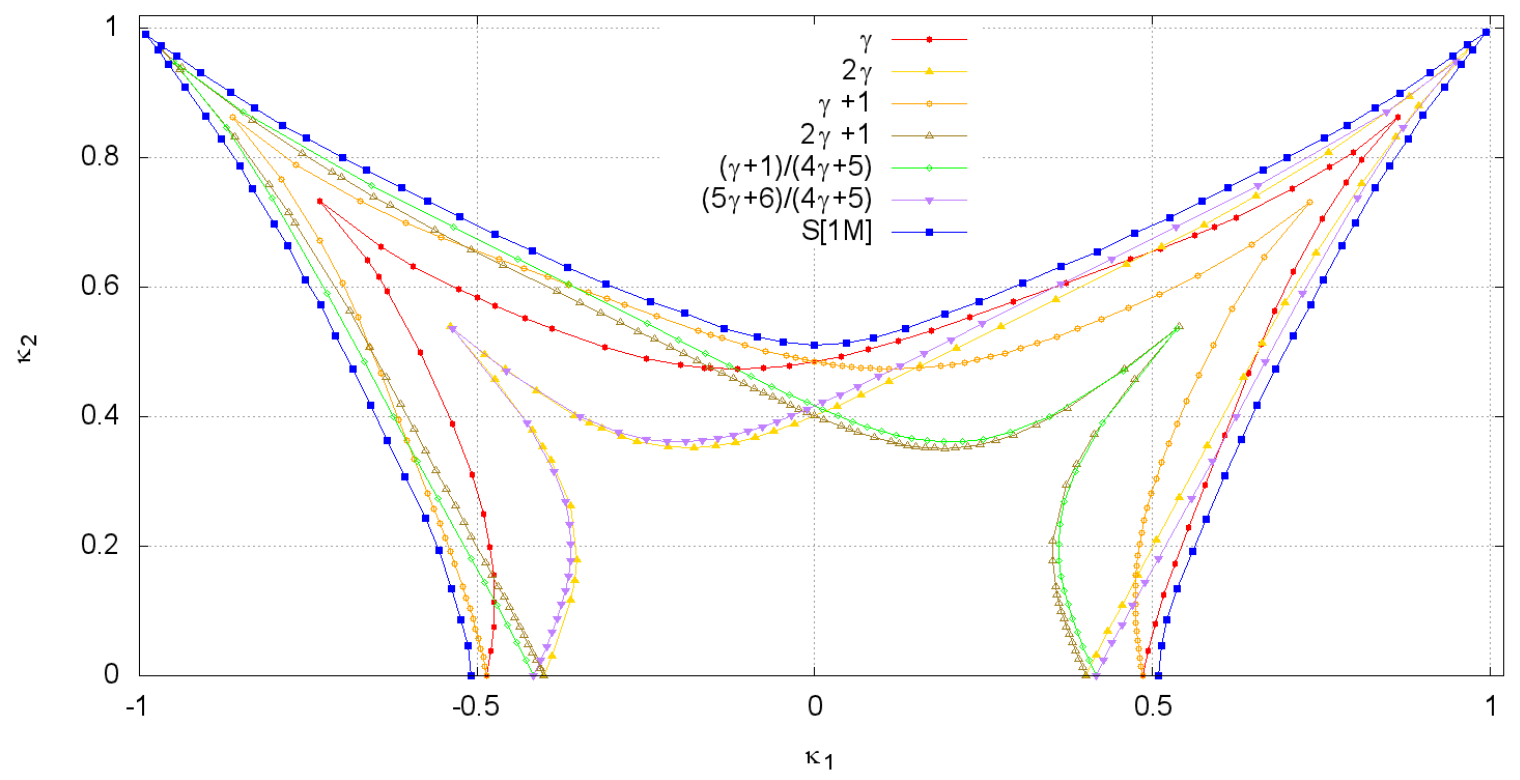

Figure 8. Critical boundaries for the existence of four KAM tori $\left(C B_{\omega}\right)\left\{\gamma, \gamma+1,2 \gamma, 2 \gamma+1, \frac{5 \gamma+6}{4 \gamma+5}\right.$ and $\left.\frac{\gamma+1}{4 \gamma+5}\right\}$ found with the parameterization method contrasted with the critical boundary $\left(C B_{g t}\right)$ found via direct simulation of the NASM.

Additionally to the rotation numbers that were predicted to be of importance in section VA, the parameterization method was applied to two additional ones: $\frac{5 \gamma+6}{4 \gamma+5}$ and $\frac{\gamma+1}{4 \gamma+5}$. These numbers were obtained empirically by iterating the map close to the invariant circle, obtaining a few digits by approximating the limit in (26) and then adding a tail of ones to the continued fraction. Neither of these two numbers correspond to a known reduced case of the map (4), however the results displayed on Figure 8 show that the corresponding $C B_{\omega}$ 's give reasonable lower bounds to the $C B_{g t}$ found by direct method in some regions of the parameter space. We are aware of the existence of robust numerical methods ${ }^{67,68}$ to estimate rotation numbers of invariant sets, however we were more interested in corroborating the consistency of the previous analytic and numeric results. We leave the numerical characterization of the rotation number of the most robust invariant circle as function of the 

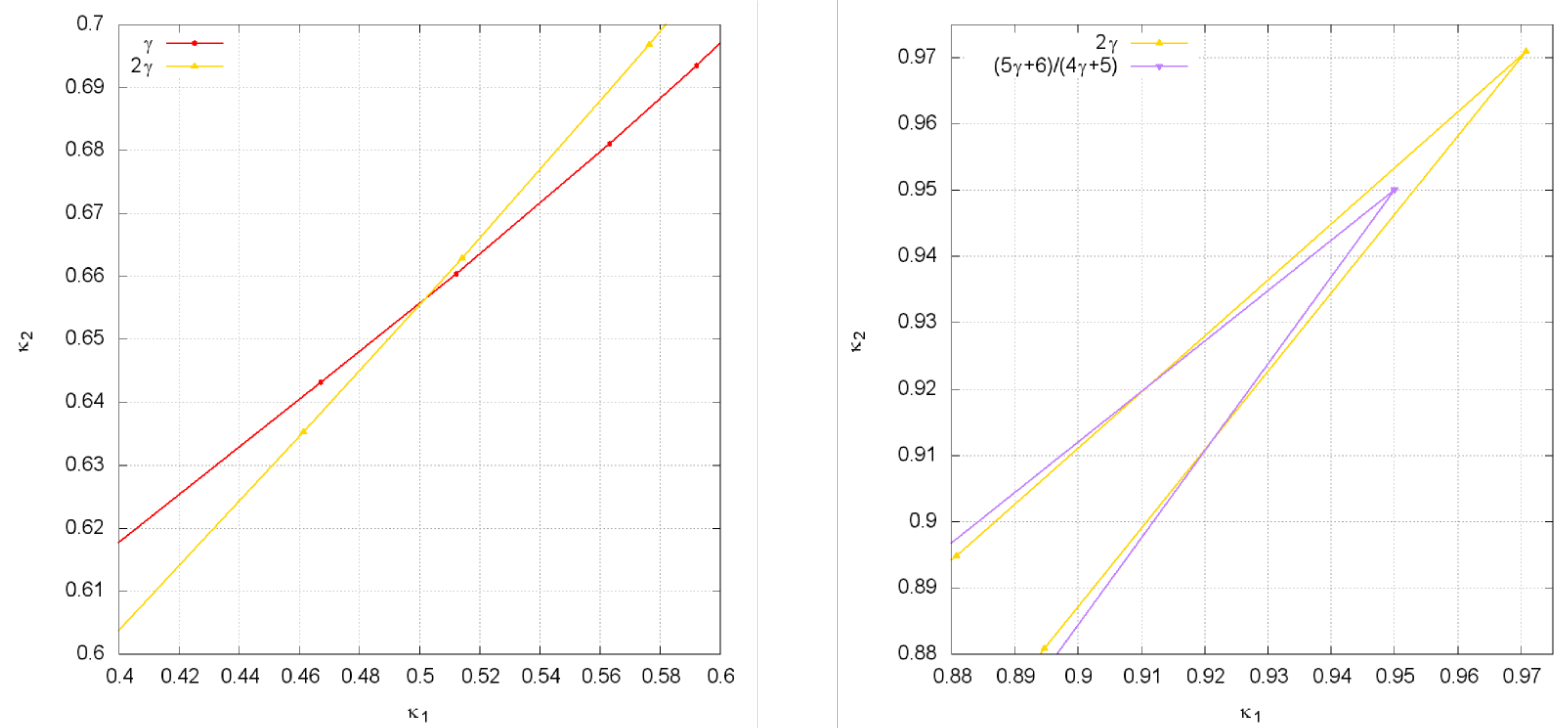

Figure 9. Details on the intersection between the critical boundaries $\left\{\gamma, 2 \gamma\right.$ and $\left.\frac{5 \gamma+6}{4 \gamma+5}\right\}$ from Fig. 8 .

parameters to a further publication.

\section{DISCUSSION AND CONCLUSIONS}

In section $\mathrm{V}$ we found from the direct computation a horn shaped critical boundary for global transport, $C B_{g t}$, giving the threshold between bounded evolution of a set of initial conditions and global transport. Using the parameterization method in Sec. VI we found the critical boundaries for the existence of invariant curves with given rotation numbers, the $C B_{\omega}$ 's. All the $C B_{\omega}$ were found to be fully contained inside the $C B_{g t}$. It is expected that the $C B_{g t}$ is minimal envelope of all the $C B_{\omega}$ associated to the invariant circles that exist for the map $\mathcal{T}_{\kappa_{1} \kappa_{2}}$.

$C B$ 's were also computed using a Greene's residue method in Ref. 42 for the $3 D$ map,

$$
\mathcal{F}_{k, \Delta k}\left(\begin{array}{c}
x \\
y \\
\phi
\end{array}\right)=\left(\begin{array}{cc}
x+y+\frac{k^{\prime}}{2 \pi} \sin (2 \pi x) & \bmod 1 \\
y+\frac{k^{\prime}}{2 \pi} \sin (2 \pi x) & \\
\phi+\Omega & \bmod 1
\end{array}\right)
$$

where $k^{\prime}=\bar{\kappa}+\Delta \kappa \cos (2 \pi \phi)$, which corresponds to a more general (quasi-periodic) variation of $\kappa$ than the one in Eq. (2). In the case $\Omega=1 / 2$, the map (56) is equivalent to the map in Eq. (1) with the appropriate choice of parameters. Note that in the special case when the initial value of $\phi$ is $1 / 4$, both maps reduce to the standard map. When the initial value of $\phi$ is 0 , the parameters of the maps are related by: $\bar{\kappa}=\frac{\kappa_{1}+\kappa_{2}}{2}, \Delta \kappa=\frac{\kappa_{2}-\kappa_{1}}{2}$. In Ref. 42 the $C B$ 
displayed correspond to the critical values of the Greene's residue ${ }^{54}$ in the parameter space $(\bar{\kappa}, \Delta \kappa)$ for map (56) for different periodic orbits with rotation vectors that approximate a two dimensional invariant torus. These $C B$ 's have a diamond shape similar to the curves obtained in Sec. V in $(\bar{\kappa}, \Delta \kappa)$ parameter variables (see Fig. 10), which represent the threshold of global transport and disappearance of all invariant one dimensional tori for map (56) with rotation vectors of the form $(\omega, 1 / 2)$. In figure 11 we compare our $C B_{g t}$ with one of the $C B$

from Ref. 42 corresponding to the periodic orbit with rotation vector $\left(\frac{1705}{3136}, \frac{2631}{3136}\right)$ with period 3136, that approximates a golden rotation vector. The critical curve is contained inside ours except for a few points that do not belong inside the region we computed.

To explore the phenomenon of the protruding regions in the parameter space that possibly exist for non autonomous maps with periods greater than two, we have studied map (56) with $\Omega=1 / 3$, which correspond to a $\kappa_{n}$ variation for map (1) with three values. Finding also critical boundaries that surpassed in a small region the one found for map (4).

Reference 43 computed the CB for a given rotation number in a driven standard map similar to Eq.(53). As in the previous case, the results are consistent with ours. In particular, they also found a diamond shaped $C B$ in the $(\bar{\kappa}, \Delta \kappa)$ parameter space.

It is important to remark that in map (56) the two dimensional and the one dimensional tori are topological barriers to global transport due the uncoupled variation of $\phi$ with respect to $(x, y)$. In general, Fig. 11 suggests that the destruction of the one dimensional tori implies the breaking of the two dimensional ones except for a few peaks that stand outside of our curve in the parameter space. It remains to study why are the two dimensional tori more robust that the one dimensional ones in these peak regions. Numerical evidence leads us to think that the critical boundary for global transport in the map (56) with $\Omega=p / q$ might fully contain the ones in Ref. 42 .

\section{ACKNOWLEDGMENTS}

We thank the referee for his valuable comments that improved the presentation of this work. This work was supported by PAPIIT IN104514 and IN110317, FENOMEC-UNAM and by the Office of Fusion Energy Sciences of the US Department of Energy at Oak Ridge National Laboratory, managed by UT-Battelle, LLC, for the U.S.Department of Energy under contract DE-AC05-00OR22725. We also express our gratitude to LUCAR-IIMAS for 


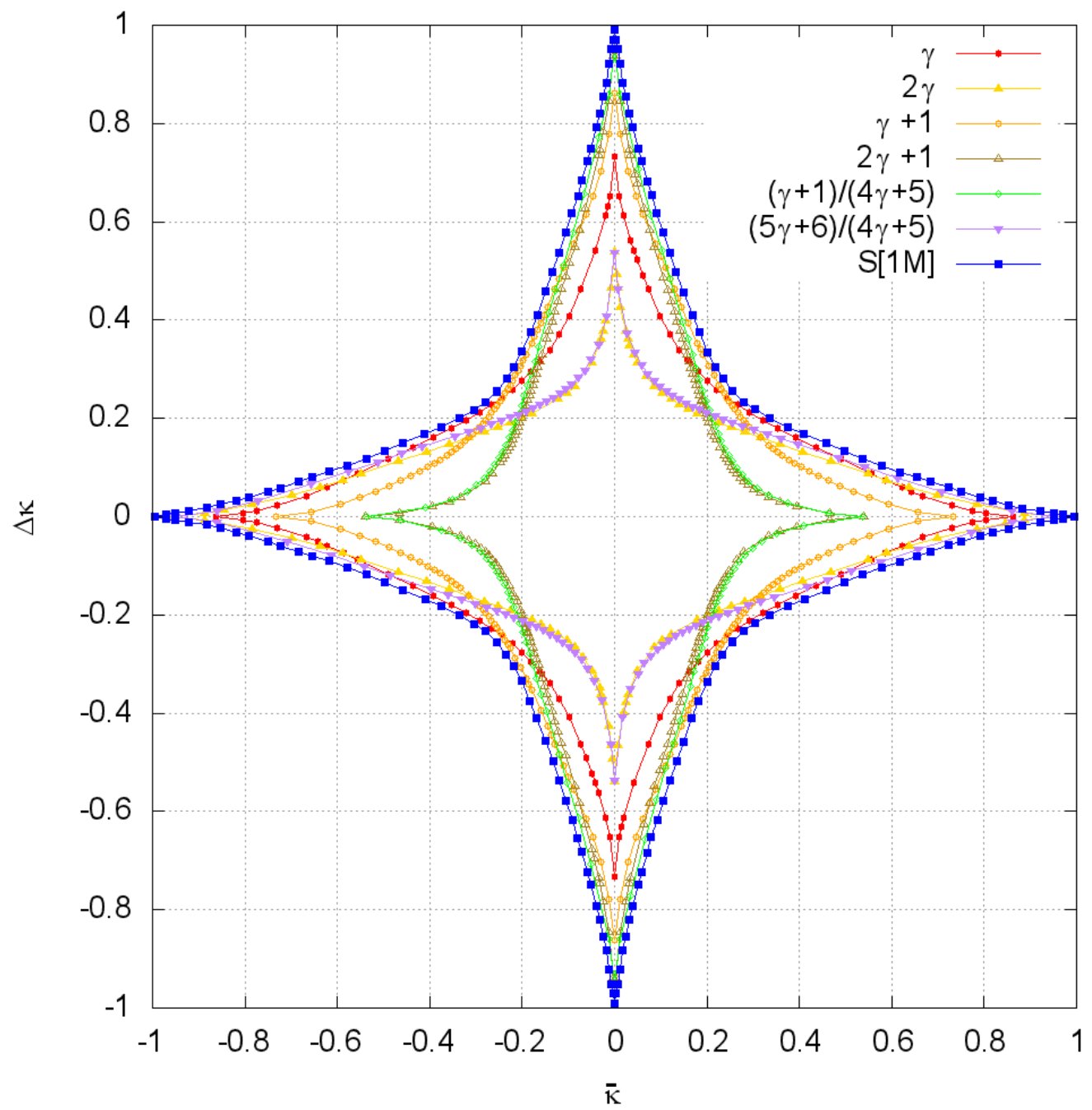

Figure 10. Plot in the $(\bar{\kappa}, \Delta \kappa)$ parameter variables of the $C B_{\omega}$ of four KAM tori $\{\gamma, \gamma+1,2 \gamma, 2 \gamma+1$, $\frac{5 \gamma+6}{4 \gamma+5}$ and $\left.\frac{\gamma+1}{4 \gamma+5}\right\}$ found with the parameterization method and the $C B_{g t}$ found via direct simulation of the NASM. The extension to the whole parameter space is obtained using the Eq. (27).

making available the CPU cluster and to the graduate program in Mathematics of UNAM for making the GPU servers available to perform our computations and especially to Ana Perez for her invaluable help.

\section{REFERENCES}

${ }^{1}$ J.D. Meiss. Symplectic maps, variational principles, and transport. Rev. Modern Phys., 64(3):795-848, 1992.

${ }^{2}$ D. Martínez, D. del Castillo-Negrete, A. Olvera, and R. Calleja. Self-consistent chaotic transport in a high dimensional mean-field hamiltonian map model. QTDS, 14(2):313-335, 


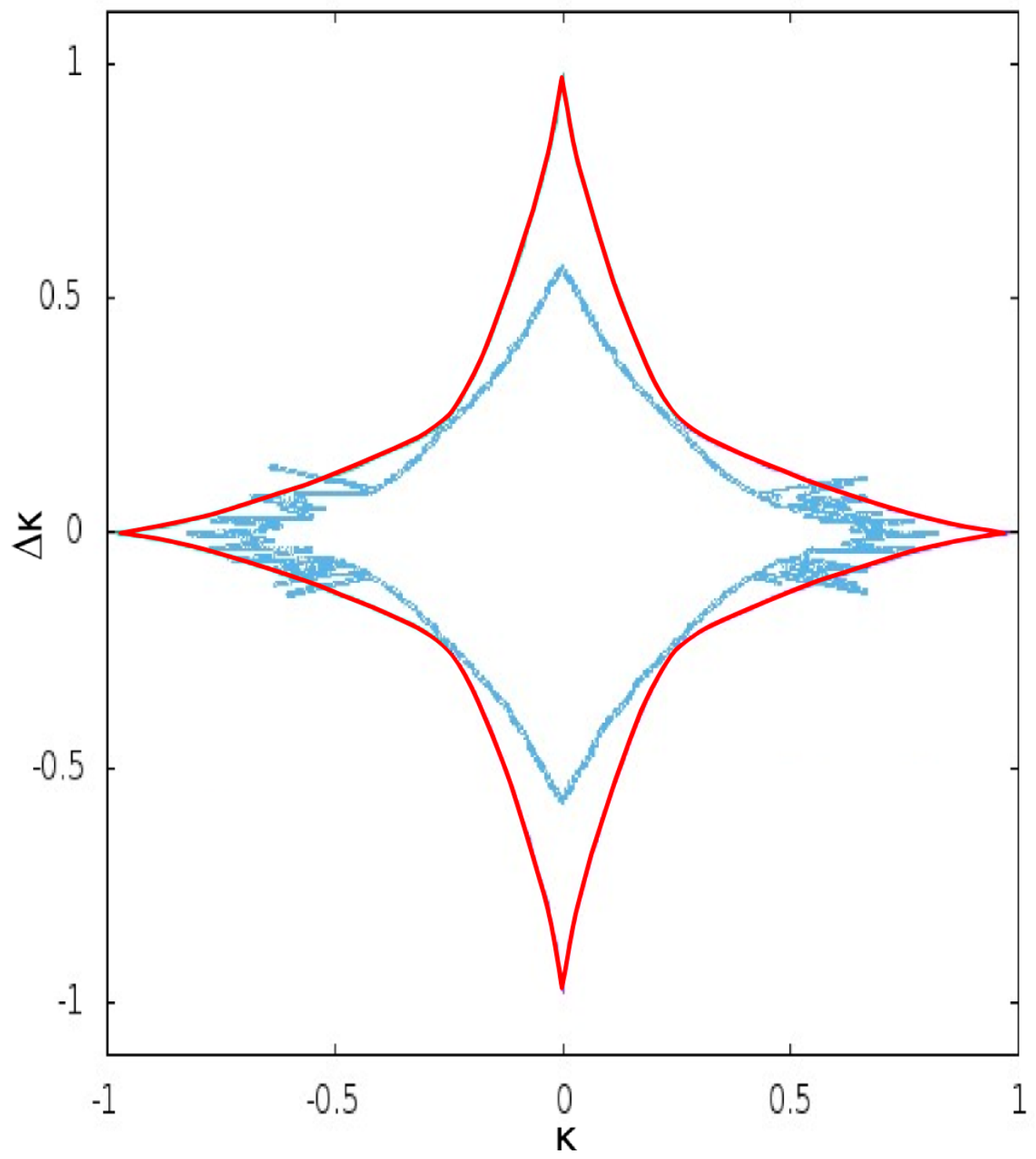

Figure 11. Critical boundary curve $\left(C B_{g t}\right)$ found via direct simulation of the NASM (red) compared with one of the $C B$ for a periodic orbit with rotation vector $\left(\frac{1705}{3136}, \frac{2631}{3136}\right)$ for map (56) (blue) taken directly from Ref. 42 [Exp. Math. 5(3):211-230,1996].

2015.

${ }^{3}$ D. del Castillo-Negrete. Self-consistent chaotic transport in fluids and plasmas. Chaos, 10(1):75-88, 2000. Chaotic kinetics and transport (New York, 1998).

${ }^{4}$ G. Bofetta, D. del Castillo, C. López, G. Pucacco, and A. Vulpiani. Diffusive transport and self-consistent dynamics in coupled maps. Phys. Rev. E, 67:026224, 2003. 
${ }^{5}$ L. Carbajal, D. del Castillo-Negrete, and J.J. Martinell. Dynamics and transport in mean-field coupled, many degrees-of-freedom, area-preserving nontwist maps. Chaos, 22(1):013137, 2012.

${ }^{6}$ D. del Castillo-Negrete. Weakly nonlinear dynamics of electrostatic perturbations in marginally stable plasmas. Physics of Plasmas, 5(11):3886-3900, 1998.

${ }^{7}$ M. Canadell and R. de la Llave. KAM tori and whiskered invariant tori for non-autonomous systems. Physica D, 310:104-113, 2015.

${ }^{8}$ S. Tompaidis. Numerical study of invariant sets of a quasiperiodic perturbation of a symplectic map. Exp. Math., 5(3):211-230, 1996.

${ }^{9}$ C. Simó. Experiments looking for theoretical predictions. Indagationes Mathematicae, 2015.

${ }^{10} \mathrm{R}$. Moeckel. Generic drift on cantor sets of annuli. In Celestial Mechanics, Dedicated to Donald Saari for his 60th Birthday, page 163, 2002.

${ }^{11}$ J.M. Greene and J.M. Mao. Higher-order fixed points of the renormalisation operator for invariant circles. Nonlinearity, 3(1):69, 1990.

${ }^{12}$ C. Baesens and R.S. MacKay. The one to two-hole transition for cantori. Physica D: Nonlinear Phenomena, 71(4):372-389, 1994.

${ }^{13} \mathrm{R}$. Calleja and R. de la Llave. Computation of the breakdown of analyticity in statistical mechanics models: numerical results and a renormalization group explanation. J. Stat. Phys., 141(6):940-951, 2010.

${ }^{14}$ R. Calleja and A. Celletti. Breakdown of invariant attractors for the dissipative standard map. Chaos, 20(1):013121, 9, 2010.

${ }^{15}$ C. Golé. Symplectic twist maps: global variational techniques, volume 18. World Scientific, 2001.

${ }^{16} \mathrm{~J}$. Moser. On invariant curves of area-preserving maps of an annulus. nach. Akad. Wiss., Gottingen, Math. Phys. Kl. II, 1(1), 1962.

${ }^{17}$ R.C. Calleja, A. Celletti, C. Falcolini, and R. de la Llave. An extension of Greene's criterion for conformally symplectic systems and a partial justification. SIAM J. Math. Anal., 46(4):2350-2384, 2014.

${ }^{18}$ J.N. Mather. Variational construction of orbits of twist diffeomorphisms. Journal of the American Mathematical Society, 4(2):207-263, 1991. 
${ }^{19}$ John N Mather. Existence of quasi-periodic orbits for twist homeomorphisms of the annulus. Topology, 21(4):457-467, 1982.

${ }^{20}$ J. M. Greene. A method for determining a stochastic transition. Journal of Mathematical Physics, 20:1183-1201, June 1979.

${ }^{21}$ B.V. Chirikov. A universal instability of many-dimensional oscillator systems. Physics reports, 52(5):263-379, 1979.

${ }^{22} \mathrm{R}$. de la Llave. A tutorial on KAM theory. In Smooth ergodic theory and its applications (Seattle, WA, 1999), volume 69 of Proc. Sympos. Pure Math., pages 175-292. Amer. Math. Soc., Providence, RI, 2001.

${ }^{23}$ R. Calleja and R. de la Llave. A numerically accessible criterion for the breakdown of quasi-periodic solutions and its rigorous justification. Nonlinearity, 23(9):2029-2058, 2010.

${ }^{24}$ R. de la Llave, A. González, À. Jorba, and J. Villanueva. KAM theory without action-angle variables. Nonlinearity, 18(2):855-895, 2005.

${ }^{25}$ R. Calleja, , A. Celletti, and R. de la Llave. A KAM theory for conformally symplectic systems: efficient algorithms and their validation. J. Differential Equations, 255(5):9781049, 2013.

${ }^{26}$ E. Zehnder. Generalized implicit function theorems with applications to some small divisor problems. I. Comm. Pure Appl. Math., 28:91-140, 1975.

${ }^{27}$ R. Calleja and R. de la Llave. Fast numerical computation of quasi-periodic equilibrium states in 1D statistical mechanics, including twist maps. Nonlinearity, 22(6):1311-1336, 2009 .

${ }^{28}$ G. Huguet, R. de la Llave, and Y. Sire. Computation of whiskered invariant tori and their associated manifolds: new fast algorithms. Discrete Contin. Dyn. Syst., 32(4):1309-1353, 2012 .

${ }^{29}$ J.-Ll. Figueras, A. Haro, and A. Luque. Rigorous computer assisted application of KAM theory: a modern approach. ArXiv e-prints, January 2016.

${ }^{30}$ À. Haro, M. Canadell, J.-Ll. Figueras, A. Luque, and J.-M. Mondelo. The parameterization method for invariant manifolds. From rigorous results to effective computations. Springer, 2016.

${ }^{31}$ R. Calleja and J.-Ll. Figueras. Collision of invariant bundles of quasi-periodic attractors in the dissipative standard map. Chaos, 22(3):033114, 10, 2012. 
${ }^{32}$ A. M. Fox and J. D. Meiss. Greene's residue criterion for the breakup of invariant tori of volume-preserving maps. Phys. D, 243:45-63, 2013.

${ }^{33}$ G. Gómez, J.-M. Mondelo, and C. Simó. A collocation method for the numerical fourier analysis of quasi-periodic functions. i. numerical tests and examples. Discrete Contin. Dyn. Syst. Ser. B, 14(1):41-74, 2010.

${ }^{34}$ A. Luque and J. Villanueva. Quasi-periodic frequency analysis using averagingextrapolation methods. SIAM Journal on Applied Dynamical Systems, 13(1):1-46, 2014.

${ }^{35}$ J.D. Meiss. Symplectic maps, variational principles, and transport. Rev. Modern Phys., 64(3):795-848, 1992.

${ }^{36}$ D. Martínez, D. del Castillo-Negrete, A. Olvera, and R. Calleja. Self-consistent chaotic transport in a high dimensional mean-field hamiltonian map model. QTDS, 14(2):313-335, 2015.

${ }^{37}$ D. del Castillo-Negrete. Self-consistent chaotic transport in fluids and plasmas. Chaos, 10(1):75-88, 2000. Chaotic kinetics and transport (New York, 1998).

${ }^{38}$ G. Bofetta, D. del Castillo, C. López, G. Pucacco, and A. Vulpiani. Diffusive transport and self-consistent dynamics in coupled maps. Phys. Rev. E, 67:026224, 2003.

${ }^{39}$ L. Carbajal, D. del Castillo-Negrete, and J.J. Martinell. Dynamics and transport in mean-field coupled, many degrees-of-freedom, area-preserving nontwist maps. Chaos, 22(1):013137, 2012.

${ }^{40}$ D. del Castillo-Negrete. Weakly nonlinear dynamics of electrostatic perturbations in marginally stable plasmas. Physics of Plasmas, 5(11):3886-3900, 1998.

${ }^{41}$ M. Canadell and R. de la Llave. KAM tori and whiskered invariant tori for non-autonomous systems. Physica D, 310:104-113, 2015.

${ }^{42} \mathrm{~S}$. Tompaidis. Numerical study of invariant sets of a quasiperiodic perturbation of a symplectic map. Exp. Math., 5(3):211-230, 1996.

${ }^{43} \mathrm{C}$. Simó. Experiments looking for theoretical predictions. Indagationes Mathematicae, 2015.

${ }^{44} \mathrm{R}$. Moeckel. Generic drift on cantor sets of annuli. In Celestial Mechanics, Dedicated to Donald Saari for his 60th Birthday, page 163, 2002.

${ }^{45}$ J.M. Greene and J.M. Mao. Higher-order fixed points of the renormalisation operator for invariant circles. Nonlinearity, 3(1):69, 1990. 
${ }^{46}$ C. Baesens and R.S. MacKay. The one to two-hole transition for cantori. Physica D: Nonlinear Phenomena, 71(4):372-389, 1994.

${ }^{47}$ R. Calleja and R. de la Llave. Computation of the breakdown of analyticity in statistical mechanics models: numerical results and a renormalization group explanation. J. Stat. Phys., 141(6):940-951, 2010.

${ }^{48}$ R. Calleja and A. Celletti. Breakdown of invariant attractors for the dissipative standard map. Chaos, 20(1):013121, 9, 2010.

${ }^{49}$ C. Golé. Symplectic twist maps: global variational techniques, volume 18. World Scientific, 2001.

${ }^{50} \mathrm{~J}$. Moser. On invariant curves of area-preserving maps of an annulus. nach. Akad. Wiss., Gottingen, Math. Phys. Kl. II, 1(1), 1962.

${ }^{51}$ R.C. Calleja, A. Celletti, C. Falcolini, and R. de la Llave. An extension of Greene's criterion for conformally symplectic systems and a partial justification. SIAM J. Math. Anal., 46(4):2350-2384, 2014.

${ }^{52}$ J.N. Mather. Variational construction of orbits of twist diffeomorphisms. Journal of the American Mathematical Society, 4(2):207-263, 1991.

${ }^{53}$ John N Mather. Existence of quasi-periodic orbits for twist homeomorphisms of the annulus. Topology, 21(4):457-467, 1982.

${ }^{54}$ J. M. Greene. A method for determining a stochastic transition. Journal of Mathematical Physics, 20:1183-1201, June 1979.

${ }^{55}$ B.V. Chirikov. A universal instability of many-dimensional oscillator systems. Physics reports, 52(5):263-379, 1979 .

${ }^{56} \mathrm{R}$. de la Llave. A tutorial on KAM theory. In Smooth ergodic theory and its applications (Seattle, WA, 1999), volume 69 of Proc. Sympos. Pure Math., pages 175-292. Amer. Math. Soc., Providence, RI, 2001.

${ }^{57} \mathrm{R}$. Calleja and R. de la Llave. A numerically accessible criterion for the breakdown of quasi-periodic solutions and its rigorous justification. Nonlinearity, 23(9):2029-2058, 2010.

${ }^{58}$ R. de la Llave, A. González, À. Jorba, and J. Villanueva. KAM theory without action-angle variables. Nonlinearity, 18(2):855-895, 2005.

${ }^{59}$ R. Calleja, , A. Celletti, and R. de la Llave. A KAM theory for conformally symplectic systems: efficient algorithms and their validation. J. Differential Equations, 255(5):9781049, 2013. 
${ }^{60}$ E. Zehnder. Generalized implicit function theorems with applications to some small divisor problems. I. Comm. Pure Appl. Math., 28:91-140, 1975.

${ }^{61}$ R. Calleja and R. de la Llave. Fast numerical computation of quasi-periodic equilibrium states in 1D statistical mechanics, including twist maps. Nonlinearity, 22(6):1311-1336, 2009 .

${ }^{62}$ G. Huguet, R. de la Llave, and Y. Sire. Computation of whiskered invariant tori and their associated manifolds: new fast algorithms. Discrete Contin. Dyn. Syst., 32(4):1309-1353, 2012 .

${ }^{63}$ J.-Ll. Figueras, A. Haro, and A. Luque. Rigorous computer assisted application of KAM theory: a modern approach. ArXiv e-prints, January 2016.

${ }^{64}$ À. Haro, M. Canadell, J.-Ll. Figueras, A. Luque, and J.-M. Mondelo. The parameterization method for invariant manifolds. From rigorous results to effective computations. Springer, 2016.

${ }^{65}$ R. Calleja and J.-Ll. Figueras. Collision of invariant bundles of quasi-periodic attractors in the dissipative standard map. Chaos, 22(3):033114, 10, 2012.

${ }^{66}$ A. M. Fox and J. D. Meiss. Greene's residue criterion for the breakup of invariant tori of volume-preserving maps. Phys. D, 243:45-63, 2013.

${ }^{67}$ G. Gómez, J.-M. Mondelo, and C. Simó. A collocation method for the numerical fourier analysis of quasi-periodic functions. i. numerical tests and examples. Discrete Contin. Dyn. Syst. Ser. B, 14(1):41-74, 2010.

${ }^{68} \mathrm{~A}$. Luque and J. Villanueva. Quasi-periodic frequency analysis using averagingextrapolation methods. SIAM Journal on Applied Dynamical Systems, 13(1):1-46, 2014. 